\title{
Intelligent Multisensor Cooperative Localization Under Cooperative Redundancy Validation
}

\author{
Lu Yin, Member, IEEE, Qiang Ni, Senior Member, IEEE, Zhongliang Deng
}

\begin{abstract}
Localization plays a key role in Internet of Things (IoT). This paper proposes a novel intelligent cooperative multisensor localization method called Edge Cloud Cooperative Localization (ECCL) which has the range and angle observations from the neighbour nodes along with the location observations from an absolute coordinate localization system like Global Positioning System (GPS). The edge cloud structure is proposed which employs several distributed Kalman Filters (KFs) in sensor nodes edge and a centralized cooperative fusion unit in the cloud. For a robust fusion, a Cooperative Redundancy Validation (CRV) method is proposed to detect the outliers. The proposed ECCL scheme has the advantages of both distributed and centralized localization, which satisfies the needs of high reliability and high accuracy, especially when sensor nodes have limited computational resources. The simulation and experiment results show that our proposed ECCL algorithm outperforms the other schemes both in outlier detection and localization accuracy.
\end{abstract}

Index Terms-Cooperative Localization, Multisensor, Cooperative Redundancy Validation.

\section{INTRODUCTION}

$\mathbf{I}$ NTERNET of Things (IoT) is a new evolution for industrial and civil applications. It mines the potential of all kinds of sensors like industrial products, smartphones and intelligent equipments. An important property of IoT is the location of the Things, such as applications for the pedestrian/vehicle navigation, logistics management, industrial equipment monitoring and venue supervision [1]-[6]. The Global Positioning System (GPS) itself is not enough in IoT applications because in these complicated environments (such as the urban areas and the buildings), the satellite signal can be blocked sometimes that might lead to very large errors or even localization suspend [7], [8]. Thus, different types of localization systems or methods are developed to overcome the weaknesses of satellite positioning system, such as using Wireless Sensor Network (WSN) [6], [9]-[14], base-station [15]-[19], Wi-Fi/Bluetooth [1], [2], [20]-[22] and ultra-wide bandwidth (UWB) signal [3], [22][26]. Hybrid localization which fuses massive observations

This research was supported in part by the National Natural Science Foundation of China under Grant 61801041, in part by the Key Research and Development Program of China under Grant 2016YFB0502001 and in part by the Royal Society project under Grant IEC170324. (Corresponding author: Qiang Ni.)

Lu Yin is with School of Electronic Engineering, Beijing University of Posts and Telecommunications, Beijing, 100876, China, and he is also with Lancaster University, InfoLab21, U.K. e-mail: inlu_mail@bupt.edu.cn.

Qiang $\mathrm{Ni}$ is with the School of Computing and Communications, Lancaster University, InfoLab21, LA1 4WA, U.K. e-mail: q.ni@lancaster.ac.uk.

Zhongliang Deng is with the Joint Center of GNSS, Chinese Ministry of Education, School of Electronic Engineering, Beijing University of Posts and Telecommunications, Beijing, 100876, China, e-mail: dengzhl@bupt.edu.cn. measured by different signal sources could provide higher localization accuracy and stronger robustness. Therefore, hybrid localization by multisensors is popular in recent years [3], [4], [12], [16], [20], [22], [27]-[30].

Sensors which can provide the range and angle observations, known as the Time of Arrival (TOA) and Angle of Arrival (AOA), are usually used in multisensor localization [12], [26], [30], [31]. In this paper, we focus on the multisensor cooperative localization which fuses absolute location measurements and TOA-AOA observations together. The absolute location may be provided by GPS or other absolute localization systems when GPS is not available, such as the pseudolite localization system [17], the Time \& Code Division-Orthogonal Frequency Division Multiplexing (TC-OFDM) localization system [15], the Bluetooth localization system [20] and so on. Cooperative localization means joint information from collaborating nodes, including the measurements and full statistical data, is considered in the localization process. In contrast, non-cooperative means there are no information exchanges between nodes.

The cooperative localization method is usually classified into two types: distributed and centralized [32], [33]. For distributed one, every node calculates its own state locally by collecting other nodes' information. Nodes are also required to share their own information in the cooperative network. For example, distributed belief propagation based algorithms, which usually use factor graph to represent the relationship of the collaborating information, have been proposed in [3], [24], [27], [34]. For the centralized one, all information from each node is gathered in a unique process unit and corresponding positioning algorithms are used to calculate the fusion result [11], [13], [31]. The usage of either distributed or centralized method depends on the computation capacity, latency needs and the hybrid accuracy.

One of the limitation of the distributed method is the computation capacity of each node. Notice that all reachable information is gathered to one node, the computation grows much with the increasing of the visible node's amount [10], [11]. Considering the low-cost IoT sensors, it is a heavy burden to calculate locally. On the other hand, to achieve a convergence of localization accuracy, several iterations are needed in the distributed hybrid method. This is to say, iterative broadcasting and receiving between nodes are implemented which will lead to long latency [24], [27], [32]. Although the centralized method also surfers the latency problem as information exchanges between nodes and central processor 
as well ${ }^{1}$, the centralized latency is much smaller than the distributed one as the iteration is not needed.

In various IoT applications, such as the vehicle navigation, logistics management and industrial equipment monitoring, either timely local localization or complicated computation is needed to ensure high reliability and accuracy [4], [35]. So a novel cooperative localization scheme, which has the potential of high accuracy localization and a local localization ability in the traffic network, must be investigated. The emerging Edge Cloud computing gives a new perspective to solve this contradiction, which plays an important role to guarantee a fast processing on the edge and strong computing capability on the cloud [36], [37]. The edge cloud structure is dramatically suitable for multisensor cooperative localization to combine the advantages of both distributed and centralized types.

From the aspect of hybrid algorithm, most of state-of-theart localization algorithms are based on geometric estimation. Cooperative localization is often modeled as geometric optimization problems. The linear/nonlinear/weighted least squares based estimator and maximum likelihood (ML) based estimator are frequently used [12], [23], [30], [31], [38]. Convex relaxation is usually employed by transforming the nonconvex problems to convex one to find the global optimal solution [9], [11], [13], [39]. Bayes filters are good at dealing with the recursive location state estimation. Kalman filter (KF) is one of the most widely implement of Bayes filters in multisensor fusion which provides the optimal estimate while both the process and measurement noise are Gaussian [14], [20], [40]-[42]. In the non-linear cases, the derivations of KF, such as extended KF (EKF) and the unscented KF (UKF) are more suitable [28], [43], [44]. KF-based interacting multiple model (IMM) provides adaptive solutions when the sensors have multiple dynamic models [4], [21]. Besides, nonparametric approaches are employed in the non-linear and non-Gaussian conditions, such as the particle filter which uses a finite number of particles to draw the posterior [22], [25]. Similar to particle filter, nonparametric belief propagation is proposed to implement distributed belief propagation algorithm [27], [34].

Different from all other existing work, we aim to develop a more accurate and reliable intelligent cooperative localization method in this work which can assess the corresponding noise and distinguish the outliers. The proposed localization methodology, called the Edge Cloud Cooperative Localization (ECCL), combines several distributed KFs in the nodes edge and a centralized cooperative fusion unit in the cloud server together to form a fusion loop. Before centralized fusion, a cooperative noise assessment method is proposed to detect the outliers for a robust fusion. The reason we employ an edge cloud structure is that it has the advantages of strong computing and fast processing capabilities. Our system design offers a novel efficient solution to combine edge preprocessing and centralized computing with the emergence of IoT and the mobile crowd sensing.

The main contributions of this paper are as follows:

${ }^{1}$ If the node's computational resources are enough, centralized localization algorithm could also be executed in each node rather than central processor. Latency between the nodes and the central processor is vanished in this kind of applications.
1) We propose a novel hybrid distributed and centralized cooperative fusion and localization framework based on the edge cloud structure to obtain a more accurate and reliable location. The proposed ECCL methodology fuses all absolute and relative measurements as well as statistical data together. It has the advantage of high capacity for huge amount sensors' fusion especially considering sensor nodes usually have limited computational resources.

2) We propose a location information validation method to detect the outliers and derive the probability of false dismissal/alarm. Our proposed methodology, called the Cooperative Redundancy Validation (CRV), considers all of the available observations. Meanwhile, it will not be affected by the outliers in the observation set when assessing others. To the best of our knowledge, it is the first time to use the probabilities (the probabilities that the location is an outlier assessed by all reference nodes) rather than the distances (between the assessed and reference nodes) to assess the outliers, so that it avoids the interference of the large errors from the reference values. The proposed CRV method enhances the system's location accuracy and robustness substantially.

The paper is organized as follows: Section II gives an introduction of the system model and the background knowledge of Kalman filter and Dempster-Shafer Evidence Theory (D$\mathrm{S}$ Theory) which will be improved in our system. Section III describes the proposed intelligent multisensor cooperative localization scheme based on the edge cloud structure. Section IV proposes the CRV method to detect the outliers. Section V evaluates the performance of the proposed methodology via simulation, numerical analysis and semi-experiment results. Finally, the conclusions are given in Section VI.

Notations: $I, 1_{M \times N}$ and $0_{M \times N}$ represent the identity matrix, the $M \times N$ matrix of ones and zeros, respectively. The operator $\operatorname{cov}(\cdot), \otimes, \circ, \oslash,{ }^{\circ}, \operatorname{erfc}(\cdot), \mathrm{u}(\cdot), U(\cdot)$ and $\|\cdot\|$ represent the covariance, the Kronecker product, the Hadamard product, the Hadamard division, the Hadamard root, the complementary Gauss error function, the Heaviside step function, the uniform distribution and the Euclidean distance, respectively. $\operatorname{tr}(A)$ represents the trace of matrix $A$. diag $(A, B)$ means a matrix which diagonal sub-matrices are matrix $A$ and $B$, and the rest of this matrix are zeros. For a square matrix $A$, $\operatorname{diag}(A)$ represents a column matrix which elements are the diagonal elements of $A$. For a column matrix $X^{(i)}$ and set $\mathcal{N}=\{1,2, \ldots, N\}$, we denote $X^{(\mathcal{N})}=$ $\left[X^{(1) T}, \ldots, X^{(N) T}\right]^{T}, X^{(i \mathcal{N})}=\left[X^{(i 1) T}, \ldots, X^{(i N) T}\right]^{T}$ and $X^{(\mathcal{N N})}=\left[X^{(1 \mathcal{N}) T}, \ldots, X^{(N \mathcal{N}) T}\right]^{T}$, respectively. For a covariance matrix $P^{(i j)}=\operatorname{cov}\left(X^{(i)}, X^{(j)}\right)$, we denote $P^{(\mathcal{N})}=$ $\left(P^{(i j)}\right), i, j \in \mathcal{N}$, where $P^{(i j)}$ s are the elements (submatrices) of $P^{(\mathcal{N})}$. Symbols frequently used in this paper are listed in Table I.

\section{Preliminary}

\section{A. System Model}

Consider a sensor network which can provide the TOAAOA measurements in their local coordinates and the position 
TABLE I

SYMBOL DEFINITION

\begin{tabular}{|c|c|}
\hline Symbol* & Definition \\
\hline$N, \mathcal{N}$ & $N$ is the total node amount. And $\mathcal{N}=\{1,2, \ldots, N\}$. \\
\hline subscript $k$ & The time slot. \\
\hline$T$ & The time interval. \\
\hline$X^{(i)}, V^{(i)}$ & Absolute location/velocity of node $i$, respectively. \\
\hline$\Phi^{(i)}$ & The state of node $i . \Phi^{(i)}=\left[X^{(i) T}, V^{(i) T}\right]^{T}$ \\
\hline$d^{(i j)}$ & $\begin{array}{l}\text { Distance between node } i \text { and node } j \text {, measured by } \\
\text { node } i \text { (In local coordinate of node } i \text { ). }\end{array}$ \\
\hline$\varphi^{(i j)}, \alpha^{(i j)}$ & $\begin{array}{l}\text { Azimuth/elevation angle of node } j \text {, measured by } \\
\text { node } i \text { (In local coordinate of node } i \text { ), respectively. }\end{array}$ \\
\hline$L^{(i j)}$ & $\begin{array}{l}\text { The local coordinates. } \\
L^{(i j)}=\left[d^{(i j)}, \varphi^{(i j)}, \alpha^{(i j)}\right]^{T} .\end{array}$ \\
\hline$\Delta X^{(i j)}$ & $\begin{array}{l}\text { Relative coordinate between node } i \text { and node } j \text {. } \\
\Delta X^{(i j)}=\left[\Delta x^{(i j)}, \Delta y^{(i j)}, \Delta z^{(i j)}\right]^{T}\end{array}$ \\
\hline$\hat{\Phi}^{(i)}$ & The prediction of node $i$ 's state $\Phi^{(i)}$ \\
\hline$\breve{\Phi}^{(i)}$ & Estimate of node $i$ 's state $\Phi^{(i)}$ by KF. \\
\hline$\breve{X}^{(i)}, \breve{V}^{(i)}$ & Location/velocity components of $\breve{\Phi}^{(i)}$, respectively. \\
\hline$X_{B P}^{(i j)}$ & $\begin{array}{l}\text { Base-point Propagation Estimate (BPE) of node } i \\
\text { calculated by node } j \text { (defined by (16)). }\end{array}$ \\
\hline$X_{C F}^{(i)}$ & Cooperative fusion result of node $i$. \\
\hline$\Phi_{F E}^{(i)}$ & Final estimate of node $i$. \\
\hline$\rho^{(i j, g)}, \rho^{(i j, 0)}$ & $\begin{array}{l}\text { Distance between the assessed and reference } \\
\text { locations (defined by (29) and (38), respectively). }\end{array}$ \\
\hline$n_{X}^{(i)}, n_{\Phi}^{(i)}$ & Measurement errors of $X_{m}^{(i)}$ and $\Phi_{m}^{(i)}$, respectively. \\
\hline $\begin{array}{l}n_{\Delta X}^{(i j)}, n_{B P}^{(i j)} \\
\quad n_{F E}^{(i)}\end{array}$ & Errors of $\Delta X_{m}^{(i j)}, X_{B P}^{(i j)}$ and $\Phi_{F E}^{(i)}$, respectively. \\
\hline$n_{P}^{(i)}$ & Process noise of node $i$. \\
\hline$n_{A, k}^{(i j)}$ & The equivalent measurement error defined by (29). \\
\hline $\begin{array}{l}n_{R}^{(i j, g)}, \\
n_{R}^{(i j, 0)}\end{array}$ & $\begin{array}{l}\text { The equivalent reference errors defined by (29) and } \\
\text { (38), respectively. }\end{array}$ \\
\hline$\sigma_{x}^{2}, \sigma_{v}^{2}$ & $\begin{array}{l}\text { Variances of the location/velocity measurement } \\
\text { errors, respectively. (Assuming the variance of each } \\
\text { node is equal to each other.) }\end{array}$ \\
\hline$\sigma_{d}^{2}, \sigma_{\varphi}^{2}, \sigma_{\alpha}^{2}$ & $\begin{array}{l}\text { Variances of the measurement errors of } d^{(i j)}, \varphi^{(i j)} \\
\text { and } \alpha^{(i j)} \text {, respectively. (Assuming the variance of } \\
\text { each node is equal to each other.) }\end{array}$ \\
\hline$\sigma_{P, x}^{2}, \sigma_{P, v}^{2}$ & $\begin{array}{l}\text { Variances of the location/velocity process noise, } \\
\text { respectively. }\end{array}$ \\
\hline$P_{P}, P_{\Phi}$ & $\begin{array}{l}\text { Covariance matrices of the process noise and the } \\
\text { measurement error, respectively. }\end{array}$ \\
\hline$P_{L}$ & Covariance matrix of $L_{m}^{(i j)}$. \\
\hline$P_{\Delta X}^{(i j)}$ & Covariance matrix of $\Delta X_{m}^{(i j)}$. \\
\hline$P_{\Delta X}^{(i-j)}$ & Covariance matrix of $\Delta X_{m}^{(i \mathcal{N})}$ and $\Delta X_{m}^{(j \mathcal{N})}$. \\
\hline$P_{K F, k \mid k-1}^{(i j)}$ & Covariance matrix of $\hat{\Phi}_{k}^{(i)}$ and $\hat{\Phi}_{k}^{(j)}$. \\
\hline$P_{K F, k}^{(i j)}$ & Covariance matrix of $\breve{\Phi}_{k}^{(i)}$ and $\breve{\Phi}_{k}^{(j)}$. \\
\hline $\begin{array}{l}P_{K F, x, k}^{(i j)} \\
P_{K F, v, k}^{(i j)}\end{array}$ & $\begin{array}{l}\text { The location/velocity component of } P_{K F, k} \text {, } \\
\text { respectively. }\end{array}$ \\
\hline$P_{B P}^{(i-j)}$ & Covariance matrix of $X_{B P}^{(i \mathcal{N})}$ and $X_{B P}^{(j \mathcal{N})}$. \\
\hline$P_{B P}^{(\mathcal{N N})}$ & $\begin{array}{l}\text { Covariance matrix of } X_{B P}^{(\mathcal{N} \mathcal{N})} \text {. Its elements } \\
\text { (sub-matrices) are } P_{B P}^{(i-j)} \mathrm{s} \text {. }\end{array}$ \\
\hline$P_{C F}^{(i j)}$ & Covariance matrix of $X_{C F}^{(i)}$ and $X_{C F}^{(j)}$. \\
\hline$P_{F E}^{(i j)}$ & Covariance matrix of $\Phi_{F E}^{(i)}$ and $\Phi_{F E}^{(j)}$. \\
\hline $\begin{array}{c}P_{A}^{(i j)}, P_{R}^{(i j, g)}, \\
P_{R}^{(i j, 0)}\end{array}$ & $\begin{array}{l}\text { Covariance matrices of } n_{A}^{(i j)}, n_{R}^{(i j, g)} \text { and } n_{R}^{(i j, 0)} \text {, } \\
\text { respectively. }\end{array}$ \\
\hline
\end{tabular}

* We use subscript $m$ to represent the measurement value in this paper i.e. $X_{m}$ represents the measurement of $X$. For saving space, measurements symbols are not listed in this table.

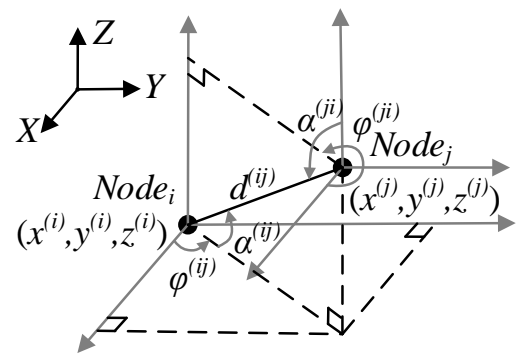

Fig. 1. System model of multisensor cooperative localization

measurements in the absolute coordinate. The system model is shown in Figure 1. For absolute coordinate, nodes could get their positions from an absolute localization system directly as:

$$
X_{m}^{(i)}=\left[\begin{array}{c}
x_{m}^{(i)} \\
y_{m}^{(i)} \\
z_{m}^{(i)}
\end{array}\right]=\left[\begin{array}{c}
x^{(i)}+n_{x}^{(i)} \\
y^{(i)}+n_{y}^{(i)} \\
z^{(i)}+n_{z}^{(i)}
\end{array}\right]=X^{(i)}+n_{X}^{(i)}
$$

Then by using the geometric relationship of the absolute and local coordinate, we can easily get the relative coordinate:

$$
\begin{aligned}
\Delta X^{(i j)} & =X^{(j)}-X^{(i)} \\
& =\left[\begin{array}{c}
d^{(i j)} \cos \left(\alpha^{(i j)}\right) \cos \left(\varphi^{(i j)}\right) \\
d^{(i j)} \cos \left(\alpha^{(i j)}\right) \sin \left(\varphi^{(i j)}\right) \\
d^{(i j)} \sin \left(\alpha^{(i j)}\right)
\end{array}\right]
\end{aligned}
$$

Note that each symbol in local coordinate has an opposite value which is measured by the other node, such as $d^{(i j)}$ and $d^{(j i)}$. Assume each pair of nodes has the same range and angle error variances, we can just average the measurement values of the two nodes. For simplification, we use superscript " $i j$ " to describe the averaged value from now on, namely:

$$
\begin{aligned}
d_{m}^{(i j)} & =\frac{1}{2}\left(d_{m}^{(i j)}+d_{m}^{(j i)}\right) \\
\varphi_{m}^{(i j)} & =\frac{1}{2}\left[\varphi_{m}^{(i j)}+\left(\varphi_{m}^{(j i)}-\pi\right)\right], i<j \\
\alpha_{m}^{(i j)} & =\frac{1}{2}\left(\alpha_{m}^{(i j)}-\alpha_{m}^{(j i)}\right)
\end{aligned}
$$

Notice that the angular measurement errors may cause $\pi$ ambiguities, the method in [30] will be used to eliminate these ambiguities in (3).

\section{B. Kalman Filter}

Consider a Markov process

$$
\Phi_{k}=A \Phi_{k-1}+n_{P, k}
$$

where $A$ is the system transition matrix. The measurement function is:

$$
\Phi_{m, k}=C \Phi_{k}+n_{\Phi, k}
$$

where $C$ is the measurement matrix. If $n_{P}$ and $n_{\Phi}$ are Gaussian noises and the initial PDF $p\left(\Phi_{0}\right)$ is Gaussian distribution, $\mathrm{KF}$ can be used to solve this problem as shown in (6)-(10) [42]:

$$
\text { Prediction: } \hat{\Phi}_{k}=A \breve{\Phi}_{k-1}
$$




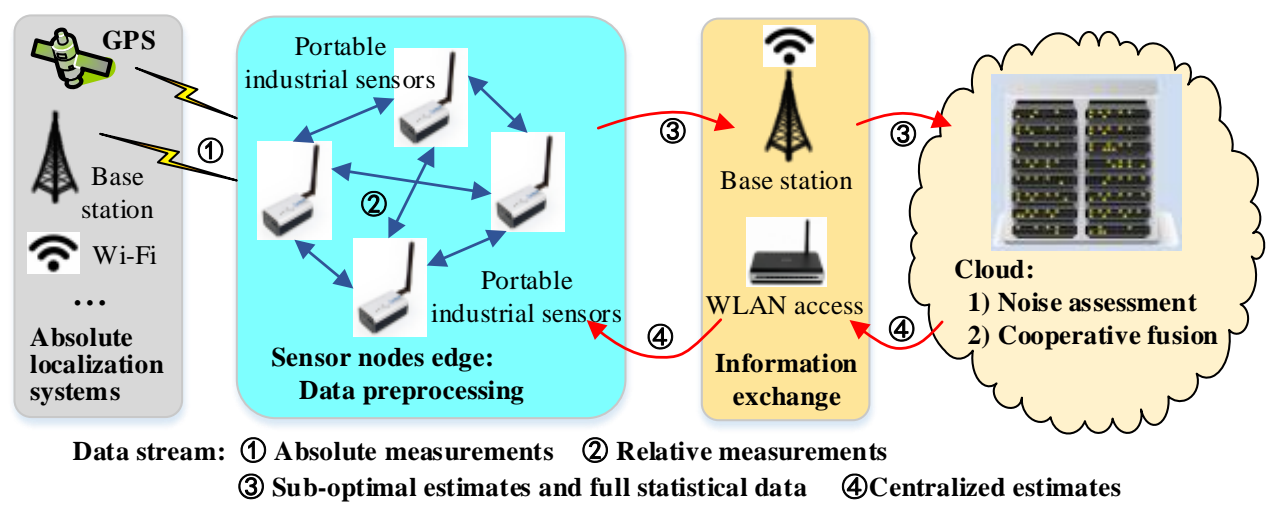

Fig. 2. Intelligent multisensor cooperative localization scheme based on edge cloud structure

$$
\text { Prediction Covariance: } P_{K F, k \mid k-1}=A P_{K F, k-1} A^{T}+P_{P}
$$

Kalman Gain: $K_{k}=P_{K F, k \mid k-1} C^{T}\left(C P_{K F, k \mid k-1} C^{T}+P_{\Phi}\right)^{-1}$

$$
\text { Optimal Estimate: } \breve{\Phi}_{k}=\hat{\Phi}_{k}+K_{k}\left(\Phi_{m, k}-C \hat{\Phi}_{k}\right)
$$

$$
\text { Update Covariance: } P_{K F, k}=\left(I-K_{k} C\right) P_{K F, k \mid k-1}
$$

\section{Dempster-Shafer (D-S) Evidence Theory}

In the D-S evidence theory [45], the frame of discernment $\Theta$ is a set of mutually exclusive and complete hypotheses. For any hypothesis $\Omega \subseteq \Theta$, it is subjected to a probability that satisfies:

$$
\begin{aligned}
m(\emptyset) & =0 \\
\sum_{\Omega \in 2^{\Theta}} m(\Omega) & =1
\end{aligned}
$$

where $0 \leq m \leq 1$ is the basic belief assignment function, $2^{\Theta}$ is the set of the subsets of $\Theta$. The belief function of $\Omega$ means the credibility that hypothesis $\Omega$ is true:

$$
\operatorname{bel}(\Omega)=\sum_{\Lambda \subseteq \Omega} m(\Lambda)
$$

where $\Lambda$ is the complete subset of $\Omega$. If there are two basic belief assignment functions $m_{1}$ and $m_{2}$, they can be fused by the Dempster's rule to yield a new basic belief assignment function $m_{12}=m_{1} \oplus m_{2}$ as:

$$
m_{12}(\Omega)=\left\{\begin{array}{cc}
0 & \Omega=\emptyset \\
\sum_{\Lambda \cap \Upsilon=\Omega} m_{1}(\Lambda) m_{2}(\Upsilon) / \mathcal{L} & \Omega \neq \emptyset
\end{array}\right.
$$

where $\mathcal{L}=\sum_{\Lambda \cap \Upsilon \neq \emptyset} m_{1}(\Lambda) m_{2}(\Upsilon)$ is the normalization coefficient. If there are more than two evidences, repeat the algorithm by using the formal fused $m_{12}$ and the new evidence's $m_{i}$ to yield the new basic belief assignment function $m_{1: i}=m_{1} \oplus m_{2} \oplus \cdots \oplus m_{i}$. For all $\Omega \in 2^{\Theta}$, the most likely hypothesis is $\hat{\Omega}=\arg \max _{\Omega}\left[m_{1: i}(\Omega)\right]$.

\section{Proposed Intelligent Multisensor COOPERATIVE Localization SCHEME}

The proposed localization scheme is based on the edge cloud structure with three phases under the assumption that all measurements are synchronous as shown in Figure 2.

1) Data preprocessing. This phase is operated in the sensor nodes edge. For absolute measurements, a pre-filter is used with the priors to obtain the state estimate which is called suboptimal estimate in this paper as it does not consider the relative measurements.

2) Noise assessment. Mulitsensor provides a lot of redundant information which will be more likely to introduce outliers than single sensor outputs. Thus, a noise assessment method is applied before the cooperative fusion. This phase is operated in the cloud.

3) Cooperative fusion. A node's location is fused with all available absolute and relative measurements, including the absolute measurement itself and the other absolute/relative measurements by cooperative fusion in the cloud. To improve the localization accuracy, the centralized estimate is fed back to the sensor nodes edge.

\section{A. Pre-filtering}

Distributed KFs are used in pre-filtering for absolute measurements of each node. The state space $\phi$ is defined in Table I. We assume each node has the same process and measurement model. Then, the process model is:

$$
A=\left[\begin{array}{llllll}
1 & 0 & 0 & T & 0 & 0 \\
0 & 1 & 0 & 0 & T & 0 \\
0 & 0 & 1 & 0 & 0 & T \\
0 & 0 & 0 & 1 & 0 & 0 \\
0 & 0 & 0 & 0 & 1 & 0 \\
0 & 0 & 0 & 0 & 0 & 1
\end{array}\right]
$$

with the covariance of process noise $P_{P}=$ $\operatorname{diag}\left(\sigma_{P, x}^{2} I_{3 \times 3}, \sigma_{P, v}^{2} I_{3 \times 3}\right)$. The position and velocity are directly output by absolute localization system, thus the measurement matrix is $C=I_{6 \times 6}$ with the covariance of measurement noise $P_{\Phi}=\operatorname{diag}\left(\sigma_{x}^{2} I_{3 \times 3}, \sigma_{v}^{2} I_{3 \times 3}\right)$.

When $k \geq 2$, the final estimate $\left(\Phi_{F E}^{(i)}\right)$ from the following cooperative fusion procedure will be fed back to the prefiltering input as $\Phi_{k}^{(i)}=\Phi_{F E, k-1}^{(i)}$. With the prior process and 


$$
J_{k}^{(i j)} \approx\left[\begin{array}{ccc}
\cos \alpha_{m, k}^{(i j)} \cos \varphi_{m, k}^{(i j)} & -d_{m, k}^{(i j)} \cos \alpha_{m, k}^{(i j)} \sin \varphi_{m, k}^{(i j)} & -d_{m, k}^{(i j)} \sin \alpha_{m, k}^{(i j)} \cos \varphi_{m, k}^{(i j)} \\
\cos \alpha_{m, k}^{(i j)} \sin \varphi_{m, k}^{(i j)} & d_{m, k}^{(i j)} \cos \alpha_{m, k}^{(i j)} \cos \varphi_{m, k}^{(i j)} & -d_{m, k}^{(i j)} \sin \alpha_{m, k}^{(i j)} \sin \varphi_{m, k}^{(i j)} \\
\sin \alpha_{m, k}^{(i j)} & 0 & d_{m, k}^{(i j)} \cos \alpha_{m, k}^{(i j)}
\end{array}\right]
$$

measurement noise covariances $P_{P}$ and $P_{\Phi}$, the error covariance reduces to $P_{K F, k}^{(i i)}=\left(I-K_{k}^{(i)}\right)\left(A P_{F E, k-1}^{(i i)} A^{T}+P_{P}\right)$ (see (7) and (10)) from $P_{\Phi}$ after KF, where $P_{F E, k-1}^{(i i)}$ is the covariance matrix of $\Phi_{F E, k-1}^{(i)}$ (see (25)).

For later use, we introduce the covariance of different node's suboptimal estimate:

$$
\begin{aligned}
& P_{K F, k}^{(i j)}=\operatorname{cov}\left(\breve{\Phi}_{k}^{(i)}, \breve{\Phi}_{k}^{(j)}\right) \\
& \quad=\left(I-K_{k}^{(i)}\right) A P_{F E, k-1}^{(i j)} A^{T}\left(I-K_{k}^{(j)}\right)^{T}, i \neq j
\end{aligned}
$$

\section{B. Cooperative Fusion}

In this subsection, the cooperative fusion algorithm is introduced under the assumption that the outliers have been rejected.

Relative measurements provide much redundant information of node's location. For example, if there are $N$ nodes, $(N-1)$ redundant locations of node $i$ will be obtained with relative observations $L_{m}^{(i j)} \forall j \neq i, j \in \mathcal{N}$, and node $j$ 's location suboptimal estimate $\breve{X}^{(j)}$. Define Base-point Propagation Estimate (BPE) $X_{B P}^{(i j)}$ as the node $i$ 's estimate location calculated by node $j$ 's suboptimal estimate and the relative measurements between the two nodes:

$$
X_{B P, k}^{(i j)}=\breve{X}_{k}^{(j)}+\Delta X_{m, k}^{(i j)}, j \neq i
$$

Thus, there will be $N$ estimate locations of a single node, including a suboptimal estimate itself and $(N-1)$ BPEs. For a simple representation, note that $X_{B P, k}^{(i i)}=\breve{X}_{k}^{(i)}$ where $\Delta X_{m, k}^{(i i)}=0$.

With the errors of relative measurements, relative coordinate is approximate to Gaussian distribution with errors $n_{\Delta X, k}^{(i j)}$ :

$$
\Delta X_{m, k}^{(i j)}=\left[\begin{array}{c}
d_{m, k}^{(i j)} \cos \alpha_{m, k}^{(i j)} \cos \varphi_{m, k}^{(i j)} \\
d_{m, k}^{(i j)} \cos \alpha_{m, k}^{(i j)} \sin \varphi_{m, k}^{(i j)} \\
d_{m, k}^{(i j)} \sin \alpha_{m, k}^{(i j)}
\end{array}\right]+n_{\Delta X, k}^{(i j)}
$$

By using Taylor series and ignoring higher order terms, the covariance matrix of (17) is obtained:

$$
P_{\Delta X, k}^{(i j)}=J_{k}^{(i j)} P_{L} J_{k}^{(i j) T}
$$

where $P_{L}=\operatorname{diag}\left(\sigma_{d}^{2}, \sigma_{\varphi}^{2}, \sigma_{\alpha}^{2}\right)$ and $J_{k}^{(i j)}$ is the Jacobi matrix of $\Delta X_{m, k}^{(i j)}$ shown in (19). Please notice $P_{\Delta X, k}^{(i i)}=0$ as $\Delta X_{m, k}^{(i i)}=0$.

We note $X_{B P}^{(i \mathcal{N})}$ as the set of all BPEs for node $i$. Because the suboptimal and relative measurements are two independent
Gaussian variable sets, the covariance matrix of different BPE sets is:

$$
\begin{aligned}
& P_{B P, k}^{(i-j)}=\operatorname{cov}\left(X_{B P, k}^{(i \mathcal{N})}, X_{B P, k}^{(j \mathcal{N})}\right) \\
& =\underbrace{\operatorname{cov}\left(\breve{X}_{k}^{(\mathcal{N})}, \breve{X}_{k}^{(\mathcal{N})}\right)}_{P_{K F, x, k}^{(\mathcal{N})}}+\underbrace{\operatorname{cov}\left(\Delta X_{k}^{(i \mathcal{N})}, \Delta X_{k}^{(j \mathcal{N})}\right)}_{P_{\Delta X, k}^{(i-j)}}
\end{aligned}
$$

The elements in $P_{\Delta X, k}^{(i-j)}$ are related to $i$ and $j$. When $i=j$, $P_{\Delta X, k}^{(i-j)}$ is a diagonal matrix (the diagonal elements are submatrices) as it is an auto-correlation of $\Delta X_{m, k}^{(i \mathcal{N})}$. When $i \neq j$, most of the elements are 0 s except the elements (sub-matrices) of $g=j$ and $h=i$ as $\left|\Delta X_{m, k}^{(i g)}\right|=\left|\Delta X_{m, k}^{(g i)}\right|=\left|\Delta X_{m, k}^{(j h)}\right|=$ $\left|\Delta X_{m, k}^{(h j)}\right|$ (see (3)). So the elements (sub-matrices) of $P_{\Delta X, k}^{(i-j)}$ are calculated as:

$$
P_{\Delta X, k}^{(i-j, g h)}= \begin{cases}P_{\Delta X, k}^{(i g)} & , i=j \text { and } g=h \\ J_{k}^{(h g)} \bar{P}_{L} J_{k}^{(g h) T} & , i \neq j \text { and } g=j \text { and } h=i \\ 0 & , \text { others }\end{cases}
$$

where $\bar{P}_{L}=\operatorname{diag}\left(\sigma_{d}^{2}, \sigma_{\varphi}^{2},-\sigma_{\alpha}^{2}\right)$ as the measurement errors of the elevation angle between two nodes are opposite after using (3). Please notice the difference between $P_{\Delta X, k}^{(i-j)}$ and $P_{\Delta X, k}^{(i j)}$.

We denote $\Psi=I_{N} \otimes 1_{N \times 1} \otimes I_{3 \times 3}$ which satisfies $X_{B P, k}^{(\mathcal{N})}=$ $\Psi X_{k}^{(\mathcal{N})}+n_{B P, k}^{(\mathcal{N})}$. Then the cooperative fusion is applied under the maximum likelihood criterion as:

$$
X_{C F, k}^{(\mathcal{N})}=\beta_{k} X_{B P, k}^{(\mathcal{N N})}
$$

where $\beta_{k}=\arg \min \left\{\operatorname{tr}\left[\operatorname{cov}\left(X_{C F, k}^{(\mathcal{N})}, X_{C F, k}^{(\mathcal{N})}\right)\right]\right\}$ is the weights calculated by the ML criterion which is obtained as:

$$
\beta_{k}=\left[\Psi^{T}\left(P_{B P, k}^{(\mathcal{N N})}\right)^{-1} \Psi\right]^{-1} \Psi^{T}\left(P_{B P, k}^{(\mathcal{N N})}\right)^{-1}
$$

The covariance matrix of $X_{C F, k}^{(\mathcal{N})}$ is:

$$
P_{C F, k}^{(\mathcal{N})}=\beta_{k} P_{B P, k}^{(\mathcal{N N})} \beta_{k}^{T}
$$

Notice that only the location is fused in the cooperative fusion, so the velocity comes from the suboptimal estimate is used as the final estimate which is defined as $\Phi_{F E, k}^{(i)}=$ $\left[X_{C F, k}^{(i) T}, \breve{V}_{k}^{(i) T}\right]^{T}$. Then, the covariance matrix of $\Phi_{F E, k}^{(i)}$ is:

$$
P_{F E, k}^{(i j)} \approx \operatorname{diag}\left(P_{C F, k}^{(i j)}, P_{K F, v, k}^{(i j)}\right)
$$

The cross-correlation between $X_{C F, k}^{(i)}$ and $\breve{V}_{k}^{(j)}$ is ignored in (25). The reasons are: 1) The variance of velocity is often much smaller than the one of location. For example, in GPS, 
the variance of velocity is about $(0.2 \mathrm{~m} / \mathrm{s})^{2}$ while the one of location is about (several meters) ${ }^{2}$. 2) The suboptimal estimate is only a part of $X_{C F, k}^{(i)}$ after cooperative fusion procedure which will weaken the cross-correlation as well.

At last, the final estimate $\Phi_{F E, k}^{(\mathcal{N})}$ and $P_{F E, k}^{(\mathcal{N})}$ will be fed back to the pre-filtering unit.

\section{LOCALIZATION INFORMATION VALIDATION}

\section{A. The Cooperative Redundancy Validation Theory}

In this section, we propose a Cooperative Redundancy Validation (CRV) method to assess the location information validation. For a standard normal Gaussian variable $\varepsilon$, if $|\varepsilon|>\Gamma$, where $\Gamma$ is a threshold determined by the significance level, $\varepsilon$ is an outlier. We denote $\xi_{0} \sim N\left(\xi, \sigma_{\xi_{0}}^{2}\right)$ as the location to be assessed where $\xi$ is the true location and $\xi_{i} \sim N\left(\xi, \sigma_{\xi_{i}}^{2}\right)$ as the reference location. If $\xi_{0}$ and $\xi_{i}$ are independent, then $\rho_{i}=\xi_{0}-\xi_{i} \sim N\left(0, \sigma_{\rho_{i}}^{2}\right)$, where $\sigma_{\rho_{i}}^{2}=\sigma_{\xi_{0}}^{2}+\sigma_{\xi_{i}}^{2}$. In the traditional validation method, if $\left|\rho_{i} / \sigma_{\rho_{i}}\right|>\Gamma, \rho_{i}$ will be rejected as an outlier. Notice that the reference value is also a Gaussian variable which might affect the assessment result, the traditional method usually has a large probability of false dismissal (PFD).

The probability that the location is an outlier is:

$$
\begin{aligned}
p_{1, i} & =p\left(\left|\frac{\xi_{0}-\xi}{\sigma_{\xi_{0}}}\right|>\Gamma \mid \rho_{i}=\xi_{0}-\xi_{i}\right) \\
& =p\left(\left|n_{\xi_{0}}\right|>\Gamma \sigma_{\xi_{0}} \mid \rho_{i}=n_{\xi_{0}}-n_{\xi_{i}}\right)
\end{aligned}
$$

where $n_{\xi_{0}}$ and $n_{\xi_{i}}$ are the errors of $\xi_{0}$ and $\xi_{i}$, respectively. We do not use the value of $\rho_{i}$ to assess the outliers directly, but to calculate the probability that $\xi_{0}$ is an outlier by $\rho_{i}$ as (26) shows. Because $\left(n_{\xi_{0}}, n_{\xi_{i}}\right) \sim N\left(0, \sigma_{\xi_{0}}^{2}, 0, \sigma_{\xi_{i}}^{2}, 0\right)$, we have:

$$
p_{1, i}=\frac{1}{2}\left[\operatorname{erfc}\left(\frac{\mu_{i}+\Gamma \sigma_{\xi_{0}}}{\sqrt{2} \sigma_{\xi_{0 i}}}\right)+\operatorname{erfc}\left(\frac{-\mu_{i}+\Gamma \sigma_{\xi_{0}}}{\sqrt{2} \sigma_{\xi_{0 i}}}\right)\right]
$$

where $\mu_{i}$ and $\sigma_{\xi_{0 i}}$ are given in Appendix A.

On the contrary, the probability that the location is not an outlier is $p_{0, i}=1-p_{1, i}$. Notice that if a location is assessed as an outlier when $p_{1, i}>p_{0, i}$, the PFD will be $p_{1, i}$ when $p_{1, i}<p_{0, i}$. Like the distributed detection problem in [46], a tolerant threshold $T_{h}$, which is determined by the desired PFD and PFA (probability of false alarm, see Section V-B), is used. When $p_{1, i}>T_{h}$, the location will be assessed as an outlier.

Normally, the variance of the reference node should be smaller than the assessed one as the ruler must be exact itself. So, we will not consider the probabilities obtained by the reference node which variance is bigger than the assessed one's.

$p_{1, i}$ and $p_{0, i}$ can be seen as the evidences that claim a location is or isn't an outlier, then D-S theory is modified and employed to fuse all probabilities to assess whether the location is an outlier. Denote $1 / 0$ as the location is/isn't an outlier respectively. The frame of discernment is $\Theta=\{0,1\}$. And then let us define the basic belief assignment vectors as $M_{i}=$ $\left[m_{i}(\Omega=\{0\})=p_{0, i}, m_{i}(\Omega=\{1\})=p_{1, i}\right], i=1,2, \ldots, N$.
Meanwhile, define the judgment vector $\mathscr{T}_{h}=\left[T_{h},\left(1-T_{h}\right)\right]$ which should be folded as $M_{i}$ s are updated by the evidences fusing process. So the modified D-S theory is developed to fuse the evidences and make the assessment decision as Algorithm 1 shows.

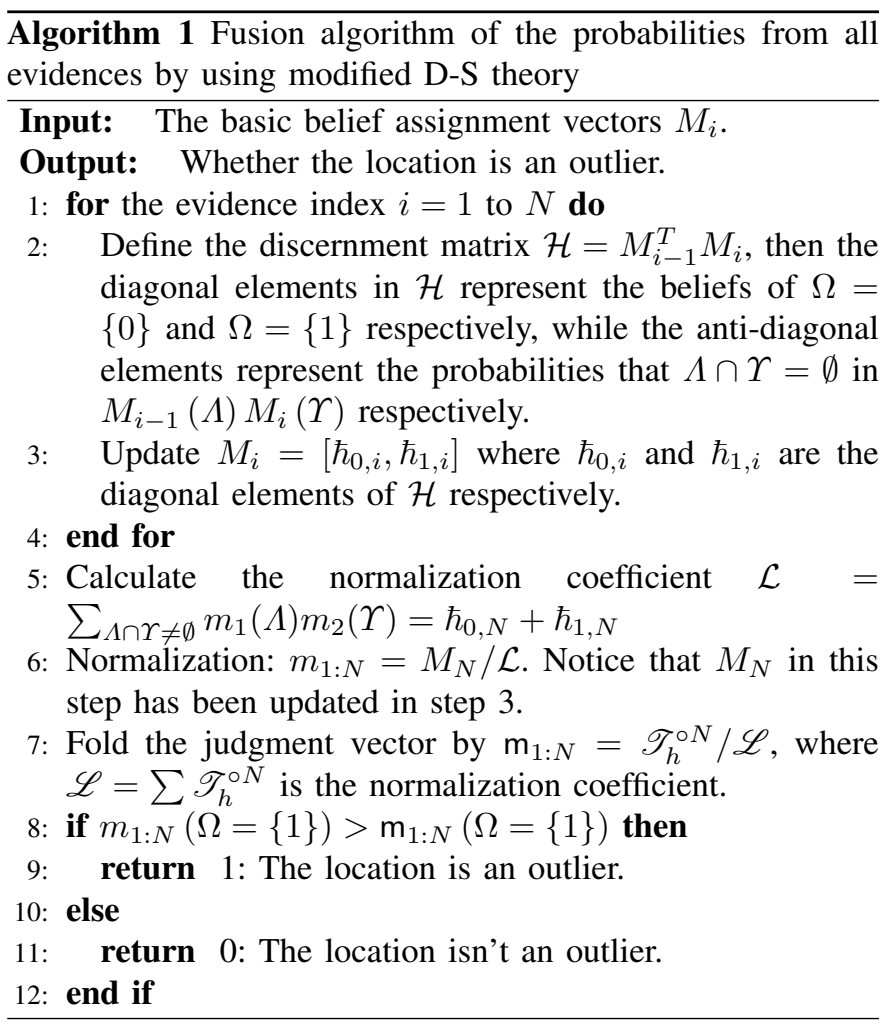

The proposed CRV method uses the probabilities rather than the distances to assess the outliers so that it avoids the interference of the large errors from the reference value. That means if the reference value has a large error, $\rho_{i}$ may be large too even if the measured location is not an outlier. Because the maximum probability is bounded to 1 , the interference of the large errors from the reference value can be much reduced when the amount of available reference nodes is not too little under the assumption that most references are non-outliers.

\section{B. Assessment of BPEs by $C R V$}

We assume $X_{B P, k}^{(i j)}$ is the assessed BPE, then we can get $N$ probabilities that claim it is an outlier $(N-1$ by the other BPEs and 1 by the prediction). Take (6) and (9) in the pre-filtering procedure to (16), then we have:

$$
X_{B P, k}^{(i j)}=\left[\left(I-K_{k}^{(j)}\right) A \Phi_{F E, k-1}^{(j)}+K_{k}^{(j)} \Phi_{m, k}^{(j)}\right]_{x}+\Delta X_{m, k}^{(i j)}
$$

where subscript $x$ represents the location component.

1) The reference is other BPEs: If $X_{B P, k}^{(i g)}, g \neq j$, is the reference BPE, the distance between the assessed and reference BPEs is shown in (29). Notice that $n_{A, k}^{(i j)}$ includes all input errors of the assessed BPE, so we denote it as the equivalent measurement error which will be assessed. $n_{A, k}^{(i j)}$ and $n_{R, k}^{(i j, g)}$ obey Gaussian distributions of mean 0 and covariances shown in (30) and (31), respectively. 


$$
\begin{aligned}
& \rho_{k}^{(i j, g)}= X_{B P, k}^{(i j)}-X_{B P, k}^{(i g)} \\
&= \underbrace{\left[\left(I-K_{k}^{(j)}\right) n_{P, k}^{(j)}+K_{k}^{(j)} n_{\Phi, k}^{(j)}\right]_{x}+n_{\Delta X, k}^{(i j)}}_{n_{A, k}^{(i j)}}+\underbrace{\left[\left(I-K_{k}^{(j)}\right) A n_{F E, k-1}^{(j)}-\left(I-K_{k}^{(g)}\right) A n_{F E, k-1}^{(g)}\right]_{x}-n_{A, k}^{(i g)}}_{-n_{R, k}^{(i j, g)}} \\
& P_{A, k}^{(i j)}=\left[\left(I-K_{k}^{(j)}\right) P_{P}\left(I-K_{k}^{(j)}\right)^{T}+K_{k}^{(j)} P_{\Phi} K_{k}^{(j) T}\right]_{x}+P_{\Delta X, k}^{(i j)} \\
& P_{R, k}^{(i j, g)}=\left[\left(I-K_{k}^{(j)}\right) A P_{F E, k-1}^{(j j)} A^{T}\left(I-K_{k}^{(j)}\right)^{T}+\left(I-K_{k}^{(g)}\right) A P_{F E, k-1}^{(g g)} A^{T}\left(I-K_{k}^{(g)}\right)^{T}-\right. \\
&\left.\quad\left(I-K_{k}^{(j)}\right) A P_{F E, k-1}^{(j g)} A^{T}\left(I-K_{k}^{(g)}\right)^{T}-\left(I-K_{k}^{(g)}\right) A P_{F E, k-1}^{(g j)} A^{T}\left(I-K_{k}^{(j)}\right)^{T}\right]_{x}+P_{A, k}^{(i g)}
\end{aligned}
$$

$$
\begin{aligned}
P_{R, k}^{(i j, 0)}= & {\left[A P_{F E, k-1}^{(i i)} A^{T}+\left(I-K_{k}^{(j)}\right) A P_{F E, k-1}^{(j j)} A^{T}\left(I-K_{k}^{(j)}\right)^{T}-\right.} \\
& \left.A P_{F E, k-1}^{(i j)} A^{T}\left(I-K_{k}^{(j)}\right)^{T}-\left(I-K_{k}^{(j)}\right) A P_{F E, k-1}^{(j i)} A^{T}+P_{P}\right]_{x}
\end{aligned}
$$

Notice that $n_{A, k}^{(i j)}$ and $n_{R, k}^{(i j, g)}$ are equivalent to $n_{\xi_{0}}$ and $n_{\xi_{i}}$ in (26), respectively. The error is assessed in each direction, then we have:

$$
\begin{gathered}
\mu_{k}^{(i j, g)}=\left(r_{k}^{(i j, g)}\right)^{\circ 2} \oslash\left[\left(r_{k}^{(i j, g)}\right)^{\circ 2}+1_{3 \times 1}\right] \circ \rho_{k}^{(i j, g)} \\
\sigma_{\xi_{0 i}, k}^{(i j, g)}=r_{k}^{(i j, g)} \oslash\left[\left(r_{k}^{(i j, g)}\right)^{\circ 2}+1_{3 \times 1}\right]^{\circ \frac{1}{2}} \circ \sigma_{\xi_{0}, k}^{(i j)}
\end{gathered}
$$

where

$$
\begin{gathered}
\sigma_{\xi_{0}, k}^{(i j)}=\operatorname{diag}\left[\left(P_{A, k}^{(i j)}\right)^{\circ \frac{1}{2}}\right] \\
\sigma_{\xi_{i}, k}^{(i j, g)}=\operatorname{diag}\left[\left(P_{R, k}^{(i j, g)}\right)^{\circ \frac{1}{2}}\right] \\
r_{k}^{(i j, g)}=\sigma_{\xi_{0}, k}^{(i j)} \oslash \sigma_{\xi_{i}, k}^{(i j, g)}
\end{gathered}
$$

$p_{1, k}^{(i j, g)}$ will be obtained by taking (32) and (33) into (27) as:

$$
\begin{aligned}
p_{1, k}^{(i j, g)}= & \frac{1}{2}\left\{\operatorname{erfc}\left[\frac{1}{\sqrt{2}}\left(\mu_{k}^{(i j, g)}+\Gamma \sigma_{\xi_{0}, k}^{(i j)}\right) \oslash \sigma_{\xi_{0 i}, k}^{(i j, g)}\right]+\right. \\
& \left.\operatorname{erfc}\left[\frac{1}{\sqrt{2}}\left(-\mu_{k}^{(i j, g)}+\Gamma \sigma_{\xi_{0}, k}^{(i j)}\right) \oslash \sigma_{\xi_{0 i}, k}^{(i j, g)}\right]\right\}
\end{aligned}
$$

2) The reference is the prediction: If the prediction $\hat{X}_{k}^{(i)}$ in the pre-filtering procedure is the reference, (29) becomes:

$$
\begin{aligned}
& \rho_{k}^{(i j, 0)}=X_{B P, k}^{(i j)}-\hat{X}_{k}^{(i)}=n_{A, k}^{(i j)}+ \\
& \underbrace{\left[\left(I-K_{k}^{(j)}\right) A n_{F E, k-1}^{(j)}-A n_{F E, k-1}^{(i)}-n_{P, k}^{(i)}\right]_{x}}_{-n_{R, k}^{(i j, 0)}}
\end{aligned}
$$

where superscript 0 represents the prediction. Similar to the calculation of $P_{R, k}^{(i j, g)}$, the covariance of $n_{R, k}^{(i j, 0)}$ is shown in (39). Then we have $p_{1, k}^{(i j, 0)}$ by replacing superscript $g$ with 0 in (32)-(37).

\section{Performance Evaluation}

In this section, we evaluate our proposed ECCL by a series of simulations and experiments. Firstly, we focus on the localization performances of ECCL by comparing several other localization methods in different scenarios. Then, we analyze the performances of CRV method in theoretical and design a specific simulation to examine its effectiveness in localization procedure. At last, we did an experiment by real hardware to confirm the simulation results.

\section{A. Localization Performance}

In this subsection, we did a number of simulations by Matlab to analyze the performance of our algorithm. The simulations are with the following assumptions:

1) The simulation area is set to a $200 \mathrm{~m} \times 200 \mathrm{~m} \times 200 \mathrm{~m}$ cube;

2) The absolute and relative measurement variances of each node are equal to each other;

3) The simulation variances are: $\sigma_{x}^{2}=(8 m)^{2}, \sigma_{v}^{2}=$ $(0.2 \mathrm{~m} / \mathrm{s})^{2}, \sigma_{d}^{2}=(1 \mathrm{~m})^{2}, \sigma_{\alpha}^{2}=\sigma_{\varphi}^{2}=\left(3^{\circ}\right)^{2} ;$

4) All measurements are synchronous;

5) Only the line-of sight (LOS) scenario is considered.

Two scenarios with 100 time slots and 100 Monte Carlo runs in each scenario are considered in the simulation:

1) Motionless scenario (MLS): 2 20 nodes are randomly placed in the simulation area, respectively.

2) Random motion scenario (RMS): 10 random trajectories are generated in the simulation area and the state probabilities are: $p_{\text {straight }}=0.8, p_{\text {left }}=p_{\text {right }}=0.1$, $p_{\text {back }}=0$. The node will turn left or right if it reaches the boundary of simulation area. The farthest relative measurement distance is up to $150 \mathrm{~m}$. The velocity of each node is about $3 \mathrm{~m} / \mathrm{s}$. 


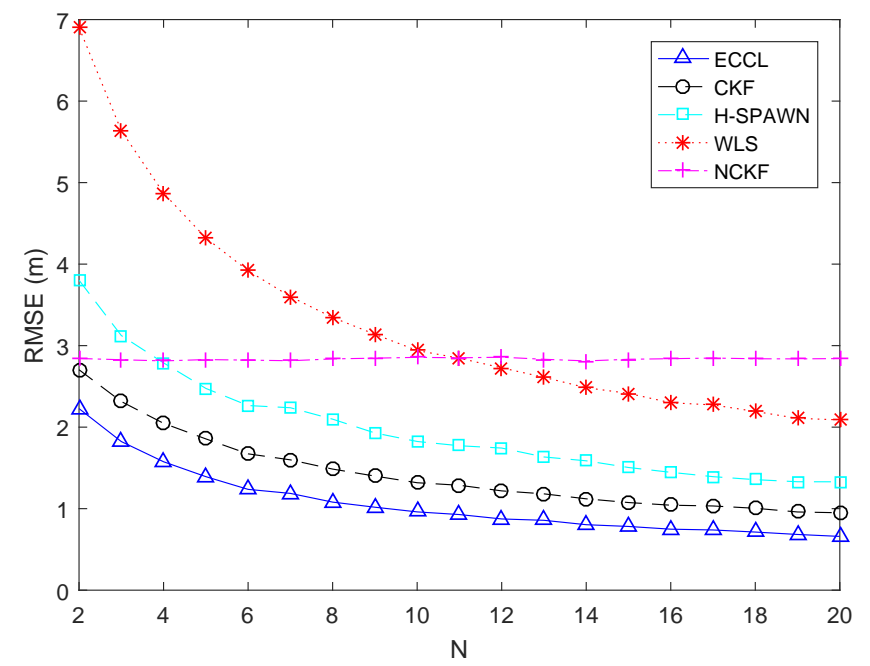

(a) RMSEs with different amount of nodes with different algorithms in MLS

Fig. 3. Location performances in different scenarios

To evaluate the performance of our ECCL localization methodology, we compared its performance with 4 other methods: the weighted least squares (WLS) [12], the cooperative fusion by KF (CKF) (filtering WLS estimates by KF), the hybrid sum-product algorithm over a wireless network (H-SPAWN) [27] and the non-cooperative localization by KF (NCKF) [42] which only considers the absolute measurements without relative measurements.

Figure 3a shows the root mean square errors $\left(\mathrm{RMSE}^{2}\right)$ when different amounts of nodes exist in MLS. It is clear that our proposed ECCL method has the highest localization accuracy than the other methods. On the other hand, the localization accuracy increases with the increasing of node amount except NCKF. And the rate of increase decreases with the increasing of node amount. Especially, there is little accuracy improvement when $N>15$ over all methods.

TABLE II

THE RMSES AND MAXIMUM ERRORS OF DIFFERENT ALGORITHMS IN RMS

\begin{tabular}{|c|c|c|c|c|c|}
\hline Algorithms & ECCL & CKF & H-SPAWN & WLS & NCKF \\
\hline \hline RMSE (m) & $\mathbf{0 . 9 8}$ & 1.34 & 1.82 & 3.05 & 2.83 \\
\hline Maximum Error $(\mathrm{m})$ & $\mathbf{4 . 1 7}$ & 4.47 & 7.33 & 9.52 & 8.71 \\
\hline
\end{tabular}

Table II shows the comparison of the RMSEs and the maximum errors ${ }^{3}$ among different algorithms in RMS. The RMSEs of the proposed ECCL methodology are $26.87 \%$, $46.15 \%, 67.87 \%$ and $65.37 \%$ smaller than the one of CKF, H-SPAWN, WLS and NCKF, respectively. And the maximum errors of proposed ECCL methodology are $6.71 \%, 43.11 \%$, $56.20 \%$ and $52.12 \%$ smaller than the one of CKF, H-SPAWN, WLS and NCKF, respectively. More detailed comparison is shown in Figure 3b. From the cumulative distribution functions

\footnotetext{
${ }^{2}$ The RMSE is calculated by the sum of squared errors in each direction.

${ }^{3}$ The location error is calculated by the Euclidean distance between the estimate and the true location.
}

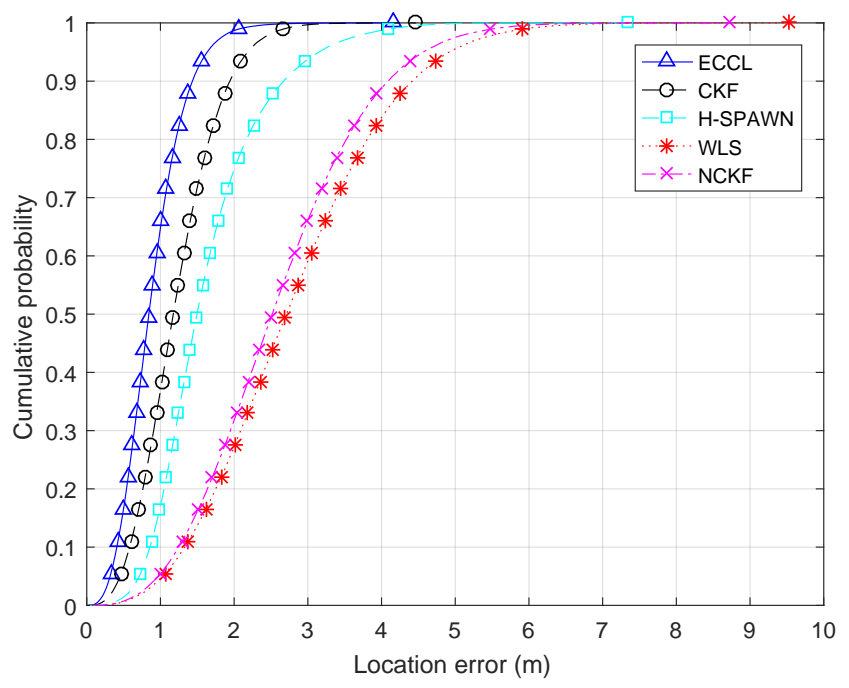

(b) The CDFs of different algorithms in RMS

(CDF), we can see more than $90 \%$ errors are under $1.5 \mathrm{~m}$ in the proposed methodology while about $2 \mathrm{~m}, 2.5 \mathrm{~m}, 4.5 \mathrm{~m}$ and $4 \mathrm{~m}$ in other methods. In cooperative localization, our methodology has the best performance among all while WLS is the worst one.

\section{B. Performance Evaluation of Cooperative Redundancy Vali- dation}

What we concern is the probability of false dismissal (PFD) and the probability of false alarm (PFA). Because the higher PFD means the higher risk of a large fusion error especially with large amount of sensor nodes. The higher PFA means more accurate location will be rejected which will lead to less accurate fusion result. In this section, the significance level is set to $10 \%$, so $\Gamma=1.65$ which is calculated by the PDF of standard normal Gaussian distribution.

1) Theoretical analysis: Firstly, we consider the condition that only one reference value exists. In this condition, The PFD/PFA of the CRV is shown in (40), where $p_{10}$ and $p_{01}$ represent the PFD and PFA respectively [Proved in Appendix B]. For saving space, we use $p_{10 / 01}$ represent the PFD and PFA in one equation respectively, and $\lesseqgtr P F D$ represents using $\leq$ when calculates PFD, while using $>$ when calculates PFA. $f_{n_{\xi_{0}}, n_{\xi_{i}}}\left(n_{\xi_{0}}, n_{\xi_{i}}\right)$ represents the joint distribution of $n_{\xi_{0}}$ and $n_{\xi_{i}}, f_{n_{\xi_{0}}}\left(n_{\xi_{0}}\right)$ represents the marginal distribution of $n_{\xi_{0}}$. Figures $4 \mathrm{a}$ and $4 \mathrm{~b}$ show the impacts of the variance ratio $r_{i 0}$ and tolerant threshold $T_{h}$ on the PFD and PFA, respectively.

We compared our method to the Mahalanobis distance method (MDM, [16]). Figure 5a shows that the PFD of CRV is better than the one of MDM, while the PFA of CRV is worse than the one of MDM when there is only 1 reference node $(N=2)$.

Then we consider the condition that more than one reference value exist. In this condition, it is hard to calculate the probabilities as the complicated process, so we did the Monte Carlo experiments to examine the performances. From Figure 


$$
\begin{aligned}
& p_{10 / 01, i}=p\left(p_{1, i} \lesseqgtr P F P D T_{h}|| n_{\xi_{0}} \mid \gtreqless P F P A \quad \Gamma \sigma_{\xi_{0}}\right) \\
& =\frac{\iint_{\left|n_{\xi_{0}}\right| \sum_{\sum P F A} \sum P \sigma_{\xi_{0}}, n_{\xi_{i}} \in R} \mathrm{u}\left( \pm_{P F D}^{P F A} p_{1, i} \mp_{P F D}^{P F A} T_{h}\right) f_{n_{\xi_{0}}, n_{\xi_{i}}}\left(n_{\xi_{0}}, n_{\xi_{i}}\right) d n_{\xi_{0}} d n_{\xi_{i}}}{\left.\int\left|n_{\xi_{0}}\right|\right|_{\sum P F A} \sum \sigma_{\xi_{0}}}
\end{aligned}
$$

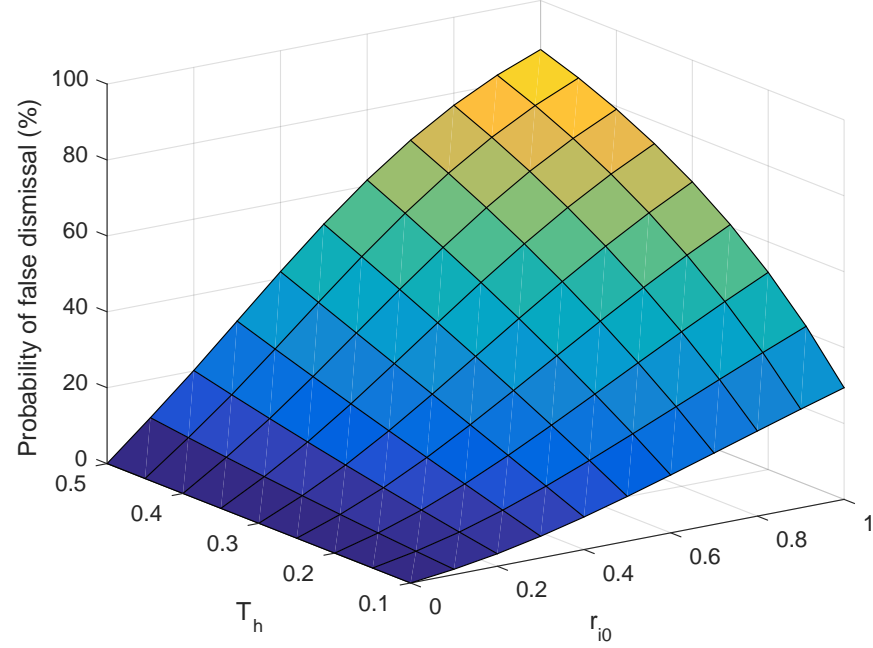

(a) Impact of $r_{i 0}$ and $T_{h}$ on the PFD

Fig. 4. Performance Evaluation that only exists one reference node

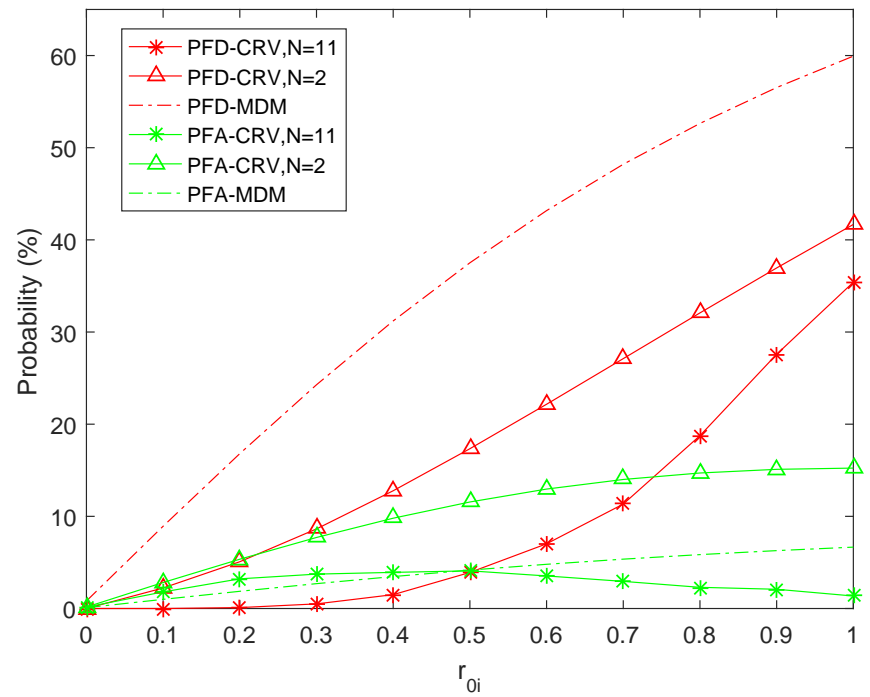

(a) Relationship between PFD/PFA and $r_{i 0}$ at different reference node amount (b)

Fig. 5. Performance Evaluation that exists multiple reference nodes

5a, we can see the PFD/PFA of CRV when $N=11$ are all better than the ones when $N=2$ and the ones of MDM. Figure $5 \mathrm{~b}$ shows the relationship between PFD/PFA and the amount of nodes when $r_{i 0}=0.5$ and $T_{h}=0.15$. It is clear that with the increasing of $N$, both of the PFD and PFA decrease.

2) The effect of $C R V$ in localization procedure: To further examine the performance of CRV, we randomly inject large outliers into the observations of RMS with a probability of $5 \%$

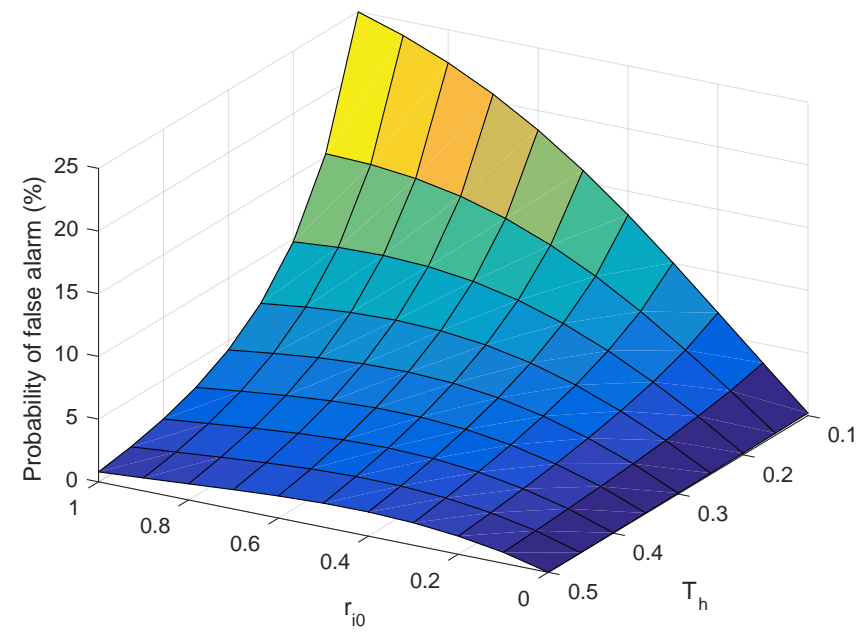

(b) Impact of $r_{i 0}$ and $T_{h}$ on the PFA

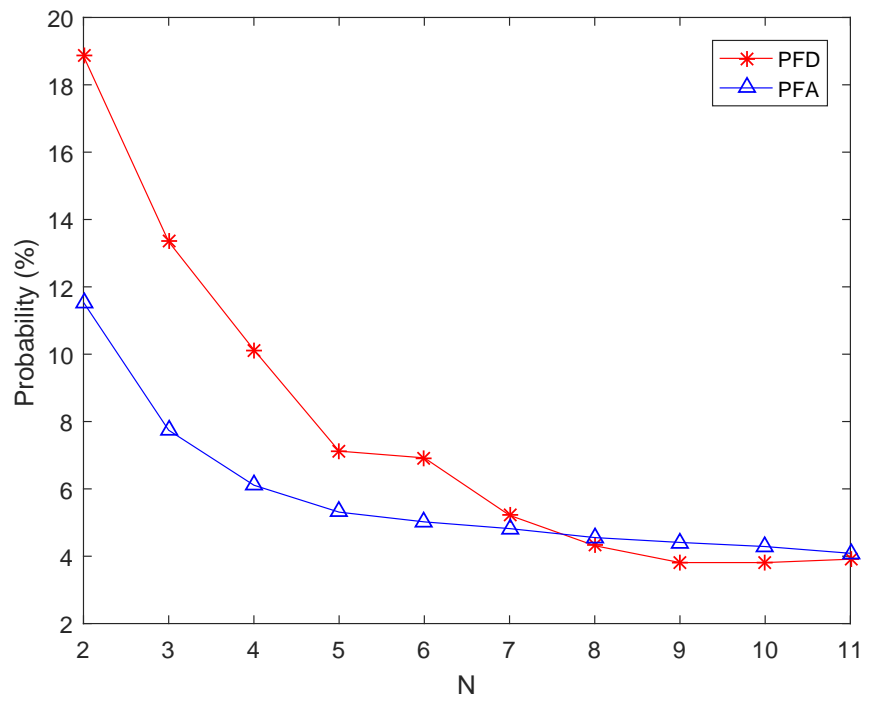

(b) Relationship between PFD/PFA and the amount of nodes when $r_{i 0}=0.5$ and $T_{h}=0.15$

to simulate the real world. The magnitudes of the additional outliers accord with $\lambda \Gamma \sigma$, where $|\lambda| \sim U(1.5,3)$ for absolute observations and $|\lambda| \sim U(1.5,5)$ for relative observations. $\sigma$ represents the standard deviation of the observation.

Denote the localization error probabilities (LEP):

$$
P_{l e}\left(e_{t h}\right)=p\left(\left\|X_{C F}-X\right\|>e_{t h}\right)
$$

where $X$ represents the true location. Figure 6 shows the LEPs 
of 4 algorithms: normal ECCL (ECCL), using MDM in ECCL instead of CRV (ECCL-CRV+MDM), ECCL without using any noise assessment method (ECCL-CRV) and the CKF. It is clear that the ECCL-CRV and CKF have more large errors as no noise assessment algorithm exists. Once an outlier appears, the final estimation will have a large bias to the true location. Then several large localization errors will follow until convergence. So not only the amount of large errors, but also the amount of small errors increases.

With comparing the curves of ECCL and ECCL$\mathrm{CRV}+\mathrm{MDM}$, we observe the latter one is close to the former one when $e_{t h}$ is small. On the contrary, the latter one moves to the other curves when $e_{t h}$ becomes larger. This is because although some outliers have been rejected by MDM which makes the amount of small errors not increase substantially, lots of outliers are dismissed which leads to more large localization errors than CRV. Of course, there are dismissed outliers by using CRV as well. But there are less dismissed outliers because of the lower PFD of CRV as Table III shows.

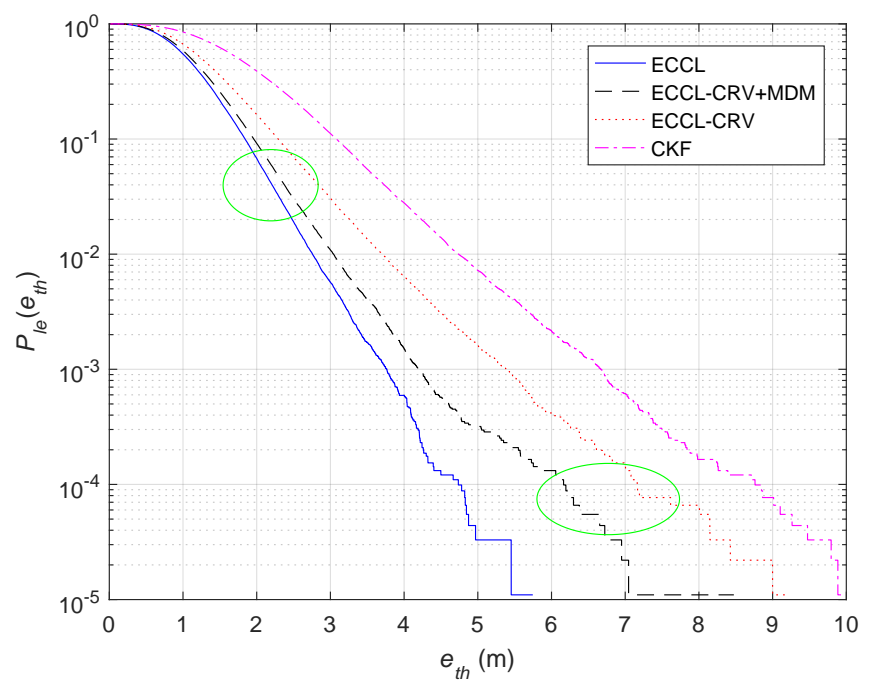

Fig. 6. Localization error probabilities

TABLE III

COMPARISON BETWEEN DIFFERENT NOISE ASSESSMENT ALGORITHMS

\begin{tabular}{|c|c|c|c|c|}
\hline Algorithms & ECCL & $\begin{array}{c}\text { ECCL-CRV } \\
\text { +MDM }\end{array}$ & ECCL-CRV & CKF \\
\hline \hline PFD (\%) & $\mathbf{1 3 . 9 4}$ & 27.74 & - & - \\
\hline PFA (\%) & $\mathbf{4 . 2 1}$ & 5.25 & - & - \\
\hline RMSE (m) & $\mathbf{1 . 2 6}$ & 1.34 & 1.55 & 2.10 \\
\hline Maximum Error (m) & $\mathbf{5 . 7 5}$ & 8.47 & 9.19 & 9.93 \\
\hline
\end{tabular}

More information could be found in Table III. We observe that the PFD of ECCL is nearly half of the one of MDM. Which confirms that there are less dismissed outliers by using CRV. Meanwhile, the PFA of ECCL is smaller than the one of MDM as well.

On the other hand, it is clear that both of the RMSE and maximum error of ECCL are much smaller than the ones of other methods, which means the proposed CRV method can distinguish most obvious outliers which lead to large localization errors. However, both of them are larger than the ones of the second column in Table II. It is the additional outliers (dismissed outliers) which make the performance reduction.

Comparing with the scenario without the additional outliers, the RMSE and maximum error of CKF deteriorate by $0.76 \mathrm{~m}$ and $5.46 \mathrm{~m}$, respectively. While these are only $0.28 \mathrm{~m}$ and $1.58 \mathrm{~m}$ for ECCL. It is because the CRV which limits the performance deterioration when outliers exist.

\section{Experiments}

In this subsection, we did a semi-experiment by real hardware to examine the simulation results. In the semiexperiment, 3 still nodes located at $(-3,0,0),(3,0,0)$ and $(0,1.5,0)$ (Unit: $\mathrm{m}$ ) in the Research Building of our campus are deployed. The absolute measurements are obtained by TC-OFDM indoor localization system [15] which accuracy is about $3 \mathrm{~m}$ ( $1 \sigma$, after filtering). The D2D range measurements with approximate $1 \mathrm{~m}(1 \sigma)$ accuracy are obtained by our selfdesigned development kit which can communicate with cloud server by Wi-Fi module. The angle measurements are simulated by adding Gaussian noises with variances $\left(3^{\circ}\right)^{2}$ to the true angles. Because the angle measurements are simulated, we say this experiment is a semi-experiment. The experiment equipments and their main parameters are shown in Figure 7 and Table IV, respectively.

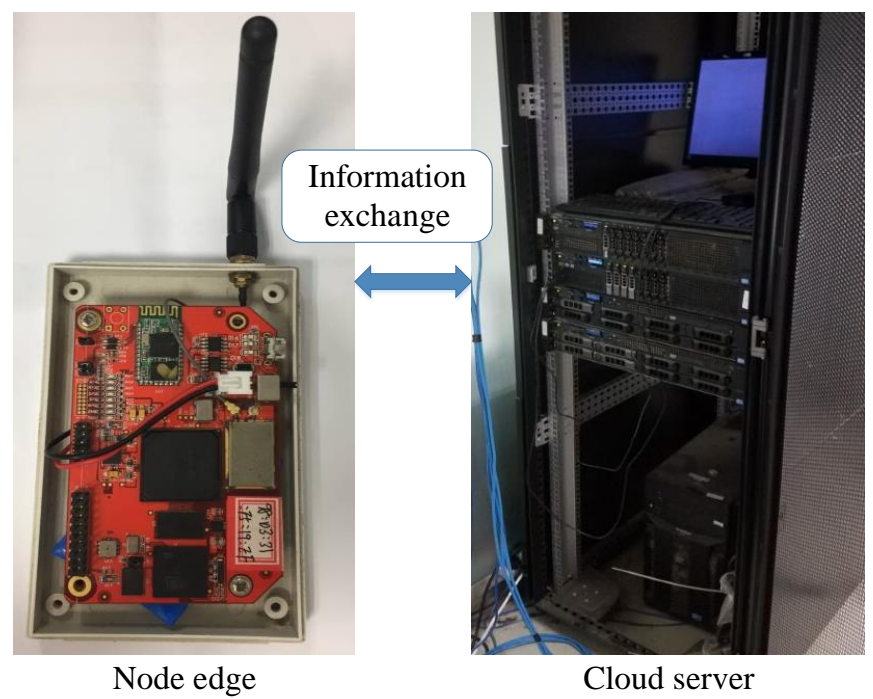

Fig. 7. Experiment equipments

For comparison, the experiment was simulated by using the Matlab simulation data whose parameters approximately subject to the real experiment. Namely, the variances of the observations approximately equal to the true ones in the experiment. And the locations of the nodes are absolutely the same as the ones in the experiment. So the simulation results can be seen as the theoretical results. The experiment 
TABLE IV

MAIN PARAMETERS OF THE EXPERIMENT EQUIPMENTS

\begin{tabular}{|c|c|c|}
\hline Equipment & Module & Parameter \\
\hline \hline \multirow{3}{*}{ Nodes edge } & CPU & $400 \mathrm{MHz}$ frequency \\
\cline { 2 - 3 } & FPGA & 80,000 logic elements \\
\cline { 2 - 3 } & Memory & $256 \mathrm{M}$ SDRAM \\
\hline \multirow{2}{*}{ Cloud server } & CPU & $2.2 \mathrm{GHz}$ with 24 cores \\
\cline { 2 - 3 } & Memory & $64 \mathrm{~GB}$ DDR III \\
\hline
\end{tabular}

last about 3 minutes with $1 \mathrm{~Hz}$ localization frequency. While the simulation last 1000 time slots.

Figure 8 shows the comparison of the CDFs between the experimental and theoretical results. We can see the experimental results are consistent with the theoretical ones well. Notice that the experimental ECCL results are slightly worse than the theoretical ones, this is mainly because the following two reasons: 1 . The variance of the experimental range measurements do not strictly equal to $(1 \mathrm{~m})^{2} ; 2$. The measurements are slightly affected by the non-line-of sight (NLOS) and there are slightly biases from the true values. Moreover, the maximum localization error of the experimental NCKF results is 2.67 times $(17.92 \mathrm{~m} / 6.73 \mathrm{~m})$ as large as the one of the theoretical results. While this proportion of ECCL results is only 1.4 times $(3.88 \mathrm{~m} / 2.83 \mathrm{~m})$. This is mainly because the experimental measurement errors are not ideal Gaussian noises and have more outliers than the simulation data as the complicated environment. And the ECCL methodology can eliminate most outliers by CRV, so the maximum error of ECCL results is not much large than the theoretical one.

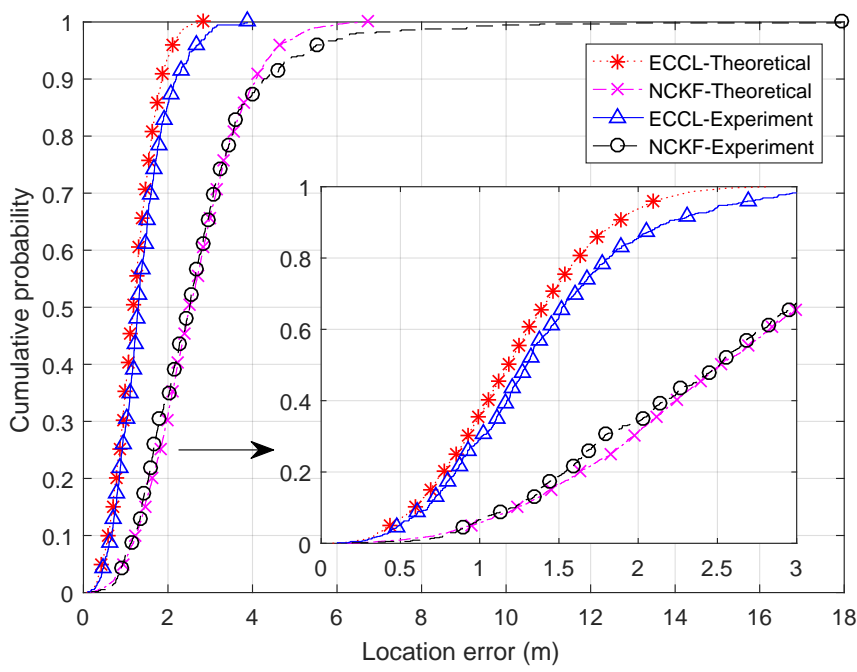

Fig. 8. The comparison of CDFs between the experimental and theoretical results

Detailed examples of the fusion process when there is or not an outlier in the semi-experiment are illustrated in Figure 9 respectively. Figure 9 is a snapshot in a time slot on the $x-y$ plane that occurs an outlier at $y$-direction of $X_{B P}^{(13)}$. The ellipses represent the uncertainties of $X_{B P}^{(13 / 12)}((13)$ in Figure 9a and (12) in Figure 9b) calculated by other reference observations which are at the center of the corresponding ellipses.
It is obvious from Figure 9a that the location at $y$-direction (the horizontal dash line through $X_{B P}^{(13)}$ ) is far from the ellipses which means all the reference observations 'think' it is an outlier. For comparison, the assessment of $X_{B P}^{(12)}$ which is not an outlier is shown in Figure 9b. Although the location at $y$ direction of $X_{B P}^{(12)}$ is far from the ellipse which center is $X_{B P}^{(13)}$ (We know this is caused by the outlier $X_{B P}^{(13)}$, but the system does not know), it is close to the other ellipses. This means only $X_{B P}^{(13)}$ 'thinks' $X_{B P}^{(12)}$ is an outlier, while both $X_{B P}^{(11)}$ (the suboptimal estimate) and the prediction 'think' it is not an outlier. So $X_{B P}^{(12)}$ is finally assessed as a non-outlier by using the proposed CRV method.

On the other hand, It is clear that the fusion location without the outlier at $y$-direction is accurate than the one with outlier. Moreover, the fusion location without the outlier at $x$-direction is more accurate than the one with outlier as well. This is because the measurement error at $\mathrm{y}$-direction will leak to $x$ direction in the cooperative fusion procedure as the crosscorrelation of each direction. Thus, the elimination of the outlier at one direction will improve the estimate accuracy at other directions as well.

\section{CONCLUSIONS}

In this paper, we focused on an accurate and robust multisensor cooperative localization method (named ECCL). A hybrid distributed and centralized fusion scheme was developed based on the edge cloud structure for strong computing capabilities and fast processing. Distributed KFs were employed for pre-filtering to obtain an suboptimal estimate which was used in the cooperative fusion. Then, to minimize the effect of the outliers, especially when there were massive observations, a localization information validation method called CRV was proposed. The simulation and semi-experiment results for the cooperative fusion and information validation were illustrated respectively. For the CRV, the PFD is significantly improved especially when two nodes have the same level of accuracy which is common in mulitsensor scenario as each node is in a similar condition. Meanwhile, the PFA reduces rapidly especially when there are massive nodes. For the localization, our ECCL has excellent performance than the other methods no matter in MLS or RMS.

The proposed ECCL methodology can be used in the industrial IoT applications, such as the vehicle localization, the security monitoring, the fire rescue and the objects tracking, etc. The edge cloud structure has the advantage of high capacity for huge amount sensors fusion especially when sensor nodes have limited computational resources.

\section{APPENDIX}

\section{A. Derivation of $p_{1, i}$}

Let's recall (26):

$$
p_{1, i}=p\left(\left|n_{\xi_{0}}\right|>\Gamma \sigma_{\xi_{0}} \mid \rho_{i}\right)=\frac{p\left(\left|n_{\xi_{0}}\right|>\Gamma \sigma_{\xi_{0}}, \rho_{i}\right)}{p\left(\rho_{i}\right)}
$$

Because $\left(n_{\xi_{0}}, n_{\xi_{i}}\right) \sim N\left(0, \sigma_{\xi_{0}}^{2}, 0, \sigma_{\xi_{i}}^{2}, 0\right)$ and $\rho_{i}=n_{\xi_{0}}-$ $n_{\xi_{i}}$, then we have (43), where $f_{n_{\xi_{0}}, n_{\xi_{i}}}\left(n_{\xi_{0}}, n_{\xi_{i}}\right)$ is the joint 


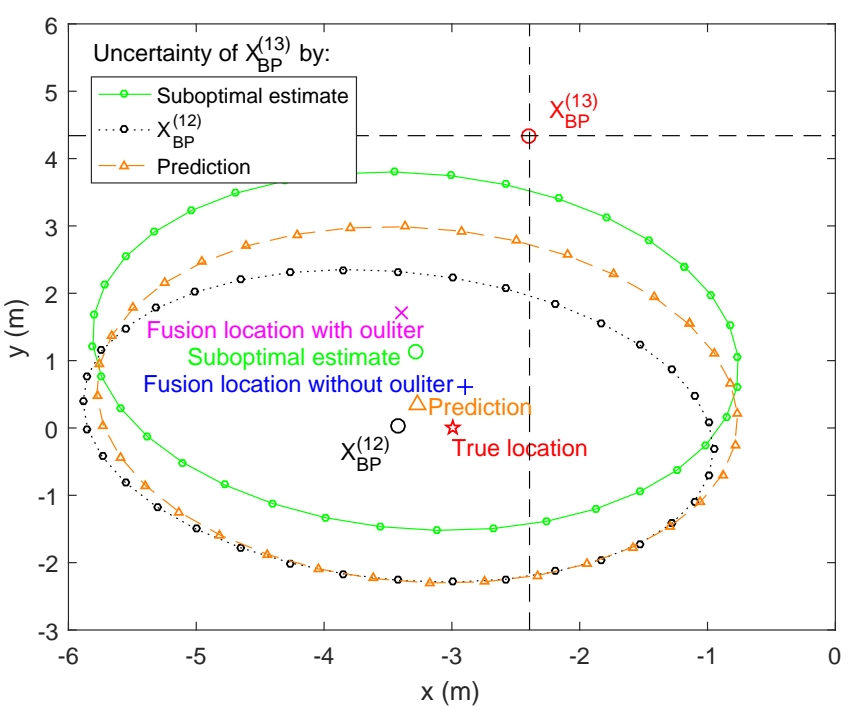

(a) The $X_{B P}^{(13)}$ in $y$-direction is an outlier

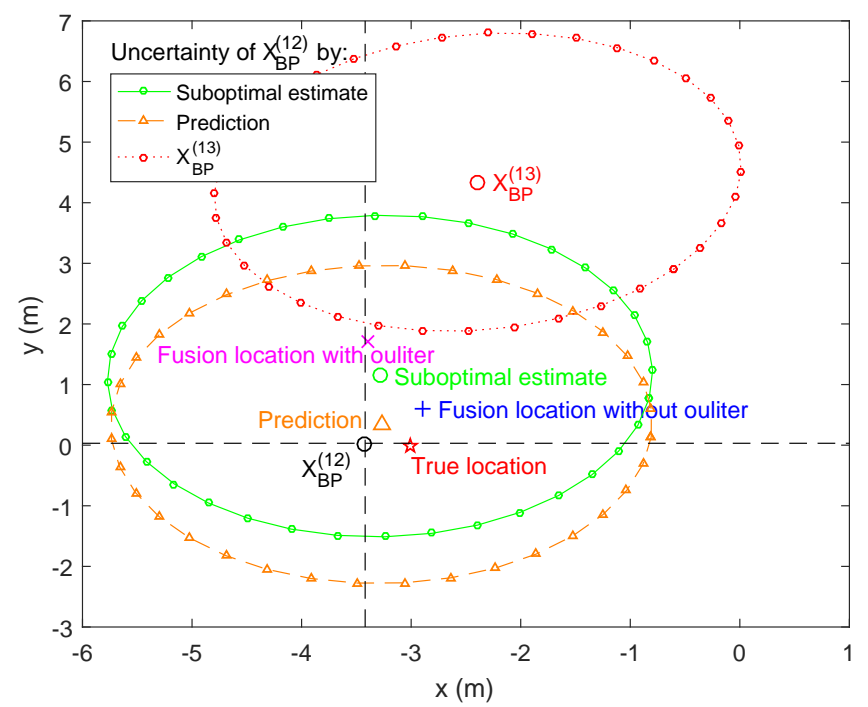

(b) The $X_{B P}^{(12)}$ in $y$-direction is not an outlier

Fig. 9. Examples of the fusion process on the $x-y$ plane

$$
\begin{gathered}
p\left(\left|n_{\xi_{0}}\right|>\Gamma \sigma_{\xi_{0}}, \rho_{i}\right)=\int_{\left|n_{\xi_{0}}\right|>\Gamma \sigma_{\xi_{0}}} f_{n_{\xi_{0}}, n_{\xi_{i}}}\left(n_{\xi_{0}}, n_{\xi_{0}}-\rho_{i}\right) d n_{\xi_{0}} \\
=\int_{\left|n_{\xi_{0}}\right|>\Gamma \sigma_{\xi_{0}}} \frac{1}{2 \pi \sigma_{\xi_{0}} \sigma_{\xi_{i}}} \exp \left\{-\frac{1}{2}\left[\frac{n_{\xi_{0}}^{2}}{\sigma_{\xi_{0}}^{2}}+\frac{\left(n_{\xi_{0}}-\rho_{i}\right)^{2}}{\sigma_{\xi_{i}}^{2}}\right]\right\} d n_{\xi_{0}} \\
p\left(\left|n_{\xi_{0}}\right|>\Gamma \sigma_{\xi_{0}}, \rho_{i}\right)=\int_{\left|n_{\xi_{0}}\right|>\Gamma \sigma_{\xi_{0}}} \frac{1}{\sqrt{2 \pi} \sigma_{\xi_{0 i}}} \exp \left[-\frac{1}{2}\left(\frac{n_{\xi_{0}}-\mu_{i}}{\sigma_{\xi_{0 i}}}\right)^{2}\right] \frac{1}{\sqrt{2 \pi} \sigma_{\rho_{i}}} \exp \left(-\frac{\rho_{i}^{2}}{2 \sigma_{\rho_{i}}^{2}}\right) d n_{\xi_{0}}
\end{gathered}
$$

distribution of $n_{\xi_{0}}$ and $n_{\xi_{i}}$. After rearrange the items we have (44), where $\mu_{i}=\frac{\sigma_{\xi_{0}}^{2}}{\sigma_{\xi_{0}}^{2}+\sigma_{\xi_{i}}^{2}} \rho_{i}, \sigma_{\xi_{0 i}}=\frac{\sigma_{\xi_{0}} \sigma_{\xi_{i}}}{\sqrt{\sigma_{\xi_{0}}^{2}+\sigma_{\xi_{i}}^{2}}}$. Notice $\rho_{i} \sim$ $N\left(0, \sigma_{\rho_{i}}^{2}\right)$, then take (44) into (42), we have:

$$
\begin{aligned}
p_{1, i} & =\int_{\left|n_{\xi_{0}}\right|>\Gamma \sigma_{\xi_{0}}} \frac{1}{\sqrt{2 \pi} \sigma_{\xi_{0 i}}} \exp \left[-\frac{1}{2}\left(\frac{n_{\xi_{0}}-\mu_{i}}{\sigma_{\xi_{0 i}}}\right)^{2}\right] d n_{\xi_{0}} \\
& =\frac{1}{2}\left[\operatorname{erfc}\left(\frac{\mu_{i}+\Gamma \sigma_{\xi_{0}}}{\sqrt{2} \sigma_{\xi_{0 i}}}\right)+\operatorname{erfc}\left(\frac{-\mu_{i}+\Gamma \sigma_{\xi_{0}}}{\sqrt{2} \sigma_{\xi_{0 i}}}\right)\right]
\end{aligned}
$$

We note $r_{i 0}=\sigma_{\xi_{i}} / \sigma_{\xi_{0}}$, then $\mu_{i}=\frac{r_{i 0}^{2}}{r_{i 0}^{2}+1} \rho_{i}$ and $\sigma_{\xi_{0 i}}=$ $\frac{r_{i 0}}{\sqrt{r_{i 0}^{2}+1}} \sigma_{\xi_{0}}$.

\section{B. Derivation of the PFD/PFA of CRV}

Taking the PFD of CRV for example, let's recall (40):

$$
\begin{aligned}
p_{10, i} & =p\left(p_{1, i} \leq T_{h}|| n_{\xi_{0}} \mid \geq \Gamma \sigma_{\xi_{0}}\right) \\
& =\frac{p\left(p_{1, i} \leq T_{h},\left|n_{\xi_{0}}\right| \geq \Gamma \sigma_{\xi_{0}}\right)}{p\left(\left|n_{\xi_{0}}\right| \geq \Gamma \sigma_{\xi_{0}}\right)}
\end{aligned}
$$

Because:

$$
\begin{aligned}
& p\left(p_{1, i} \leq T_{h},\left|n_{\xi_{0}}\right| \geq \Gamma \sigma_{\xi_{0}}\right) \\
& \quad=\int_{\left|n_{\xi_{0}}\right| \geq \Gamma \sigma_{\xi_{0}}} p\left(T_{h}-p_{1, i} \geq 0 \mid n_{\xi_{0}}\right) p\left(n_{\xi_{0}}\right) d n_{\xi_{0}}
\end{aligned}
$$

Notice that $p\left(n_{\xi_{i}} \mid n_{\xi_{0}}\right)=p\left(n_{\xi_{i}}\right)$ if $n_{\xi_{0}}$ and $n_{\xi_{i}}$ are independent, so:

$$
p\left(T_{h}-p_{1, i} \geq 0 \mid n_{\xi_{0}}\right)=\int_{-\infty}^{\infty} \mathrm{u}\left(T_{h}-p_{1, i}\right) f_{n_{\xi_{i}}}\left(n_{\xi_{i}}\right) d n_{\xi_{i}}
$$

where $f_{n_{\xi_{i}}}\left(n_{\xi_{i}}\right)$ represents the marginal distribution of $n_{\xi_{i}}$. Note $f_{n_{\xi_{0}}}\left(n_{\xi_{0}}\right)$ as the marginal distribution of $n_{\xi_{0}}$, then we have (40) by taking (47) and (48) into (46).

Similarly, we can calculate PFA of CRV by the aforementioned method.

\section{REFERENCES}

[1] X. Lu, H. Zou, H. Zhou, L. Xie, and G.-B. Huang, "Robust extreme learning machine with its application to indoor positioning," IEEE transactions on cybernetics, vol. 46, no. 1, pp. 194-205, 2016.

[2] L.-H. Chen, E. H.-K. Wu, M.-H. Jin, and G.-H. Chen, "Intelligent fusion of Wi-Fi and inertial sensor-based positioning systems for indoor pedestrian navigation," IEEE Sensors Journal, vol. 14, no. 11, pp. 4034 4042,2014 
[3] H. M. Georges, Z. Xiao, and D. Wang, "Hybrid cooperative vehicle positioning using distributed randomized sigma point belief propagation on non-Gaussian noise distribution," IEEE Sensors Journal, vol. 16, no. 21, pp. 7803-7813, 2016.

[4] K. Jo, K. Chu, and M. Sunwoo, "Interacting multiple model filterbased sensor fusion of GPS with in-vehicle sensors for real-time vehicle positioning," IEEE Transactions on Intelligent Transportation Systems, vol. 13, no. 1, pp. 329-343, 2012.

[5] D. Gulati, F. Zhang, D. Clarke, and A. Knoll, "Vehicle infrastructure cooperative localization using factor graphs," in 2016 IEEE Intelligent Vehicles Symposium (IV), 2016, pp. 1085-1090.

[6] M. Farooq-I-Azam, Q. Ni, and E. A. Ansari, "Intelligent energy efficient localization using variable range beacons in industrial wireless sensor networks," IEEE Transactions on Industrial Informatics, vol. 12, no. 6 pp. 2206-2216, 2016.

[7] A. Razavi, D. Gebre-Egziabher, and D. M. Akos, "Carrier loop architectures for tracking weak GPS signals," IEEE Transactions on Aerospace \& Electronic Systems, vol. 44, no. 2, pp. 697-710, 2008.

[8] C. Zhu and X. Fan, "A novel method to extend coherent integration for weak GPS signal acquisition," IEEE Communications Letters, vol. 19, no. 8, pp. 1343-1346, 2015.

[9] Y. Zhang, S. Xing, Y. Zhu, F. Yan, and L. Shen, "RSS-based localization in WSNs using Gaussian mixture model via semidefinite relaxation," IEEE Communications Letters, 2017.

[10] T. Lv, H. Gao, X. Li, S. Yang, and L. Hanzo, "Space-time hierarchicalgraph based cooperative localization in wireless sensor networks," IEEE Transactions on Signal Processing, vol. 64, no. 2, pp. 322-334, 2015.

[11] S. Tomic, M. Beko, and D. Rui, "RSS-based localization in wireless sensor networks using convex relaxation: Noncooperative and cooperative schemes," IEEE Transactions on Vehicular Technology, vol. 64 no. 5, pp. 2037-2050, 2014.

[12] M. W. Khan, N. Salman, and A. H. Kemp, "Optimised hybrid localisation with cooperation in wireless sensor networks," IET Signal Processing, vol. 11, no. 3, pp. 341-348, 2016.

[13] S. Tomic, M. Beko, and D. Rui, "3-D target localization in wireless sensor network using RSS and AoA measurements," IEEE Transactions on Vehicular Technology, vol. PP, no. 99, pp. 1-1, 2017.

[14] X. Fang, L. Nan, Z. Jiang, and L. Chen, "Multi-channel fingerprint localisation algorithm for wireless sensor network in multipath environment," IET Communications, vol. 11, no. 15, pp. 2253-2260, 2017.

[15] Z. Deng, Y. Yu, X. Yuan, N. Wan, and L. Yang, "Situation and development tendency of indoor positioning," China Communications, vol. 10 , no. 3, pp. $42-55,2013$.

[16] G. De Angelis, G. Baruffa, and S. Cacopardi, "GNSS/cellular hybrid positioning system for mobile users in urban scenarios," IEEE Transactions on Intelligent Transportation Systems, vol. 14, no. 1, pp. 313-321, 2013.

[17] A. Vervisch-Picois and N. Samama, "Interference mitigation in a repeater and pseudolite indoor positioning system," IEEE Journal of Selected Topics in Signal Processing, vol. 3, no. 5, pp. 810-820, 2009.

[18] A. Shahmansoori, G. E. Garcia, G. Destino, G. Seco-Granados, and $\mathrm{H}$. Wymeersch, " $5 \mathrm{G}$ position and orientation estimation through millimeter wave MIMO," in Globecom Workshops (GC Wkshps), 2015 IEEE. IEEE, 2015, pp. 1-6.

[19] A. Dammann, R. Raulefs, and S. Zhang, "On prospects of positioning in 5G," in IEEE International Conference on Communication Workshop, 2015, pp. 1207-1213

[20] Z. Deng, X. Fu, and H. Wang, "An IMU-aided body-shadowing error compensation method for indoor bluetooth positioning," Sensors, vol. 18, no. 1, p. 304, 2018

[21] S. Y. Cho, "Measurement error observer-based IMM filtering for mobile node localization using WLAN RSSI measurement," IEEE Sensors Journal, vol. 16, no. 8, pp. 2489-2499, 2016.

[22] N. Bargshady, K. Pahlavan, and N. A. Alsindi, "Hybrid WiFi/UWB, cooperative localization using particle filter," in International Conference on Computing, Networking and Communications, 2015, pp. 1055-1060.

[23] G. De Angelis, A. Moschitta, and P. Carbone, "Positioning techniques in indoor environments based on stochastic modeling of UWB round-triptime measurements," IEEE Transactions on Intelligent Transportation Systems, vol. 17, no. 8, pp. 2272-2281, 2016.

[24] H. Wymeersch, J. Lien, and M. Z. Win, "Cooperative localization in wireless networks," Proceedings of the IEEE, vol. 97, no. 2, pp. 427450, 2009.

[25] P. H. Tseng, Z. Ding, and K. T. Feng, "Cooperative self-navigation in a mixed LOS and NLOS environment," IEEE Transactions on Mobile Computing, vol. 13, no. 2, pp. 350-363, 2014
[26] B. Hanssens, D. Plets, E. Tanghe, C. Oestges, D. P. Gaillot, M. Lienard, L. Martens, and W. Joseph, "An indoor localization technique based on ultra-wideband AoD/AoA/ToA estimation," in IEEE International Symposium on Antennas and Propagation, 2016, pp. 1445-1446.

[27] M. A. Caceres, F. Penna, H. Wymeersch, and R. Garello, "Hybrid cooperative positioning based on distributed belief propagation," IEEE Journal on Selected Areas in Communications, vol. 29, no. 10, pp. 1948 1958, 2011.

[28] M. A. Caceres, F. Sottile, R. Garello, and M. A. Spirito, "Hybrid GNSSToA localization and tracking via cooperative unscented Kalman filter," in IEEE International Symposium on Personal, Indoor and Mobile Radio Communications Workshops, 2010, pp. 272-276.

[29] D. Gulati, F. Zhang, D. Clarke, and A. Knoll, "Graph-based cooperative localization using symmetric measurement equations:," Sensors, vol. 17, no. 6, p. 1422, 2017.

[30] Z. Wang and S. A. Zekavat, "A novel semidistributed localization via multinode TOA-DOA fusion," IEEE Transactions on Vehicular Technology, vol. 58, no. 7, pp. 3426-3435, 2009.

[31] K. Yu and Y. J. Guo, "Anchor global position accuracy enhancement based on data fusion," IEEE Transactions on Vehicular Technology, vol. 58, no. 3, pp. 1616-1623, 2009.

[32] Y. Shi, Q. Cui, S. Cao, X. Zhang, and X. Tao, "Performance relationship between distributed and centralised cooperative localisations," Electronics Letters, vol. 50, no. 2, pp. 127-128, 2014.

[33] L. Shu, J. Lloret, J. J. P. C. Rodrigues, and M. Chen, "Distributed intelligence and data fusion for sensor systems," IET Communications, vol. 5, no. 12, pp. 1633-1636, 2011.

[34] A. T. Ihler, J. W. Fisher, R. L. Moses, and A. S. Willsky, "Nonparametric belief propagation for self-localization of sensor networks," IEEE Journal on Selected Areas in Communications, vol. 23, no. 4, pp. 809-819, 2005.

[35] N. B. Truong, G. M. Lee, and Y. Ghamri-Doudane, "Software defined networking-based vehicular adhoc network with fog computing," in IFIP/IEEE International Symposium on Integrated Network Management, 2015, pp. 1202-1207.

[36] D. Georgakopoulos, P. P. Jayaraman, M. Fazia, M. Villari, and R. Ranjan, "Internet of things and edge cloud computing roadmap for manufacturing," IEEE Cloud Computing, vol. 3, no. 4, pp. 66-73, 2016.

[37] L. Shu, Y. Chen, Z. Huo, N. Bergmann, and L. Wang, "When mobile crowd sensing meets traditional industry," IEEE Access, 2017.

[38] H. Yu, G. Huang, and J. Gao, "Constrained total least-squares localisation algorithm using time difference of arrival and frequency difference of arrival measurements with sensor location uncertainties," IET Radar Sonar \& Navigation, vol. 6, no. 9, pp. 891-899, 2012.

[39] C. Soares, J. Xavier, and J. Gomes, "Simple and fast convex relaxation method for cooperative localization in sensor networks using range measurements," IEEE Transactions on Signal Processing, vol. 63, no. 17, pp. 4532-4543, 2015.

[40] Y. Sun, Q. Sun, and K. Chang, "The application of indoor localization systems based on the improved Kalman filtering algorithm," in International Conference on Systems and Informatics, 2018, pp. 768-772.

[41] H. Y. Chung, C. C. Hou, and Y. S. Chen, "Indoor intelligent mobile robot localization using fuzzy compensation and Kalman filter to fuse the data of gyroscope and magnetometer," IEEE Transactions on Industrial Electronics, vol. 62, no. 10, pp. 6436-6447, 2015.

[42] S. Thrun, W. Burgard, and D. Fox, Probabilistic robotics. MIT press, 2005.

[43] S. Yousefi, X. W. Chang, and B. Champagne, "Mobile localization in non-line-of-sight using constrained square-root unscented Kalman filter," IEEE Transactions on Vehicular Technology, vol. 64, no. 5, pp. 20712083, 2015.

[44] Y. Huang, Y. Zhang, B. Xu, Z. Wu, and J. Chambers, "A new adaptive extended Kalman filter for cooperative localization," IEEE Transactions on Aerospace \& Electronic Systems, vol. 54, no. 1, pp. 353-368, 2018.

[45] K. Sentz and S. Ferson, Combination of evidence in Dempster-Shafer theory. Citeseer, 2002, vol. 4015.

[46] R. Viswanathan and P. K. Varshney, "Distributed detection with multiple sensors Part I Fundamentals," Proceedings of the IEEE, vol. 85, no. 1, pp. 54-63, 1997. 


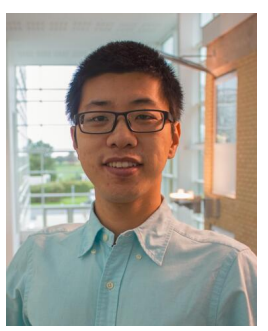

Lu Yin (M'17) received the B.Sc. and Ph.D degrees in electrical engineering from Beijing University of Posts and Telecommunications (BUPT), Beijing, China, in 2014. He is currently an assistant professor with School of Electronic Engineering, BUPT. He was an academic visitor with InfoLab21, Lancaster University from 2016 to 2017 . He is a member of the IEEE. His current research interests are in the areas of GNSS, indoor positioning, integrated/cooperative positioning, intelligent localization (localization with artificial intelligence), positioning-communication integrative system and communication system.

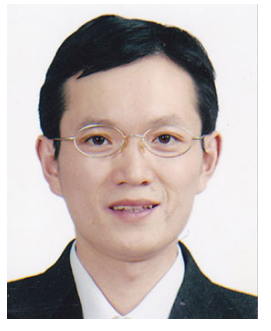

Qiang Ni (M'04-SM'08) received the B.Sc., M.Sc., and Ph.D. degrees from the Huazhong University of Science and Technology, China, all in engineering. $\mathrm{He}$ is currently a Professor and the Head of the Communication Systems Group, School of Computing and Communications, Lancaster University, InfoLab21, Lancaster, U.K. His main research interests lie in the area of future generation communications and networking, including green communications and networking, cognitive radio network systems, heterogeneous networks, small cell and ultra-dense networks, 5G and 6G, SDN, cloud networks, energy harvesting, wireless information and power transfer, IoTs and vehicular networks. He has authored or co-authored over 200 papers in these areas. He was the IEEE 802.11 Wireless Standard Working Group Voting Member and a contributor to the IEEE Wireless Standards.

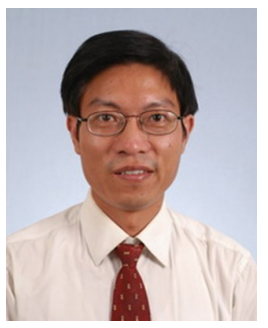

Zhongliang Deng received the MSc degree in manufacturing engineering from BeiHang University, and $\mathrm{PhD}$ degree in mechanical manufacture from Tsinghua University, China. During 2002-2003, he was the senior visiting scholar of southern California University in USA. He is a professor at School of Electronic Engineering in Beijing University of Posts and Telecommunications now. He holds the position of director of research with the Wireless Network Positioning and Communication Integration Research Center since 2006. His research interests include indoor and outdoor seamless positioning, GNSS, satellite communications, MEMS and multimedia. 